\title{
Synthesis and in-vitro antioxidant and antitumor evaluation of novel pyrazole-based heterocycles
}

\author{
Mahmoud F. Ismail $^{1}$ D . Amira A. El-sayed ${ }^{1}$
}

Received: 18 August 2018 / Accepted: 30 November 2018 / Published online: 13 December 2018

(C) Iranian Chemical Society 2018

\begin{abstract}
Hydrazide-hydrazone, namely, $N^{\prime}$-((5-chloro-3-methyl-1-phenyl-1H-pyrazol-4-yl)methylene)-2-cyanoacetohydrazide 3, was utilized as a key starting material for the construction of a variety of novel heterocyclic moieties. The newly synthesized compounds were characterized by elemental analyses and spectral data (IR, ${ }^{1} \mathrm{H}-\mathrm{NMR}$, and mass spectra). Some of the newly synthesized compounds were screened for their antitumor and antioxidant activities. The results showed clearly that compounds $\mathbf{6 , 1 1}, \mathbf{1 3}, \mathbf{2 2}$, and 29 displayed promising in-vitro antitumor activity against two cell lines (HepG2 and MCF-7). Among these compounds, compounds 13 and 29 exhibited the highest activity as antitumor agents. On the other hand, compounds 13, 22, and 29 exhibited the highest inhibitory antioxidant activity using ABTS method. While, in case of erythrocytes hemolysis, compounds 4, 13, and 29 proved to exhibit potent antioxidative activity as vitamin C. Meanwhile, compounds 6, 11, 16, 21, and 22 showed a remarkable promising activity. In light of the highest potency of our novel compounds in targeting both antitumor and antioxidant activities, compounds $\mathbf{1 3}$ and $\mathbf{2 9}$ warrant persistent preclinical development as antitumor and antioxidant agents.
\end{abstract}

\section{Graphical abstract}

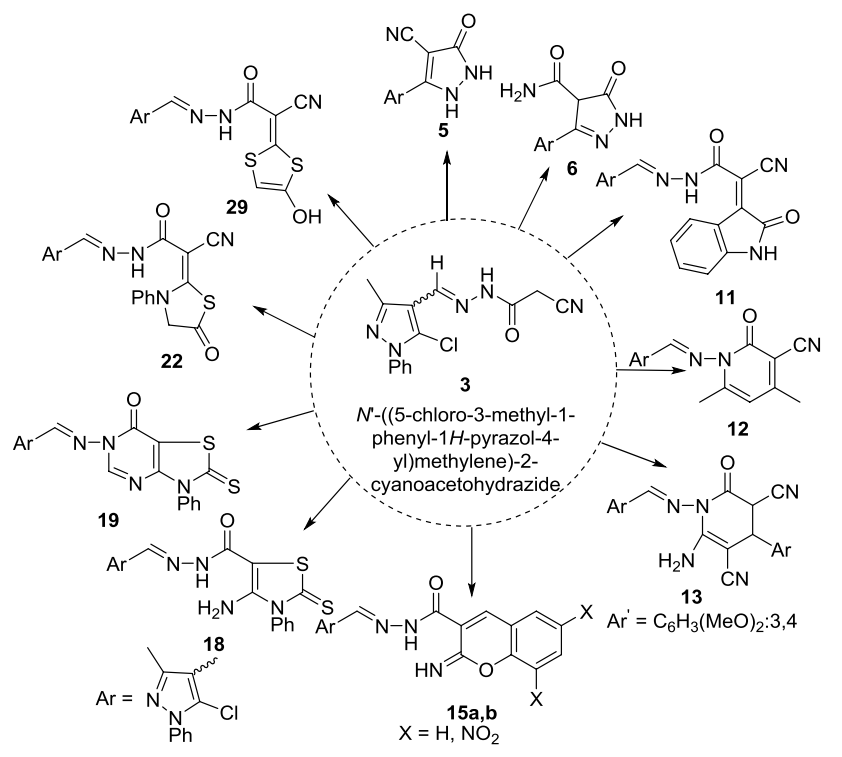

Keywords Pyrazole $\cdot$ Diarylidene hydrazine $\cdot$ Iminocoumarin $\cdot 1,3$-Thiazole $\cdot$ Antioxidant $\cdot$ Antitumor

Electronic supplementary material The online version of this article (https://doi.org/10.1007/s13738-018-1566-x) contains supplementary material, which is available to authorized users.

Extended author information available on the last page of the article 


\section{Introduction}

The pyrazole nucleus is a ubiquitous feature of a pharmacophoric scaffold, which represents a class of heterocyclic compounds with a wide range of biological applications. Many of them are widely used as potent analgesic, antiarrhythmic and anticonvulsant [1], antidiabetic [2], anticancer [3], anti-inflammatory [4, 5], anti-conceptive and antipyretic [6], muscle relaxing [7], antifungal [8, 9], antibacterial activities [8], insecticidal [10], herbicidal [11], anti HIV, antagonist of OPL1, BACE inhibitors for the treatment of Alzheimer's disease, hepatitis $\mathrm{C}$ virus, and hypoxia inducible factor [12].

After profound investigation, various methods are available for the synthesis of the pyrazole moiety such as utility of the catalytic performance of $\mathrm{Fe}_{3} \mathrm{O}_{4} @$ HNTs-PEI [13], utility of a cellulose-based nanobiocomposite decorated with $\mathrm{Fe}_{3} \mathrm{O}_{4}$ nanoparticles in water as a green solvent at ambient temperature [14], and via multicomponent reactions (MCRs) are powerful synthetic tools [15].

From synthetic aspect, since cyanoacetohydrazide derivatives possess both electrophilic and nucleophilic properties [16]. They have been used to design diverse heterocyclic moieties such as pyrazole, 1,3-dithiolane, thiadiazole, 1,3-thiazolidinone, iminocoumarin, and pyridazine derivatives [17-19].

Due to all of the aforementioned facts and in continuation our previous work, $N^{\prime}$-((5-chloro-3-methyl-1-phenyl$1 H$-pyrazol-4-yl)methylene)-2-cyanoacetohydrazide $\mathbf{3}$ as a readily available precursor for the synthesis of such new heterocyclic systems which might show pharmacological effects.

\section{Experimental}

\section{Chemistry}

\section{General information}

Melting points are uncorrected and were determined in open capillary tubes using an electric melting point apparatus (G-K). The IR spectra $\left(400-4000 \mathrm{~cm}^{-1}\right)$ were recorded on the Nicolet iS10 FT-IR spectrometer using $\mathrm{KBr}$ Wafer technique. ${ }^{1} \mathrm{H}-\mathrm{NMR}$ spectrum was recorded with a Varian Gemini spectrometer at $400 \mathrm{MHz}$ with TMS as the internal standard and chemical shifts were reported on a $\delta$ scale (ppm) using $\mathrm{CDCl}_{3}$ or DMSO- $\mathrm{d}_{6}$ as solvents, while the coupling constants ( $J$ values) are given in $\mathrm{Hz}$. Elemental analyses $(\mathrm{C}, \mathrm{H}, \mathrm{N}$, and $\mathrm{S})$ were carried out at the Microanalytical Data Centre at the Faculty of Science, Cairo University, Egypt. Mass spectra were performed on Shimadzu GC-MS QP1000EX apparatus in central analytical lab, Ain Shams University. All reactions were monitored by TLC (thin layer chromatography, Merck) and spots were detected using a UV lamp (254 nm). Biological activity was tested at the drugs department, Faculty of pharmacy, Mansoura University, Egypt.

\section{$N^{\prime}$-((5-Chloro-3-methyl-1-phenyl-1H-pyrazol-4-yl) methylene)-2-cyanoacetohydrazide 3}

A mixture of 5-chloro-3-methyl-1-phenyl-1H-pyrazole4-carbaldehyde 1 ( $2.2 \mathrm{~g}, 0.01 \mathrm{~mol})$ and cyanoacetohydrazide $2(0.99 \mathrm{~g}, 0.01 \mathrm{~mol})$ in absolute ethanol and/or dioxane $(30 \mathrm{ml})$ was heated under reflux for $4 \mathrm{~h}$. The pale yellow solid which separated after slow evaporation was collected by filtration and then recrystallized from ethanol to give 3 as pale yellow crystals; m.p.: $190{ }^{\circ} \mathrm{C}$, yield: $87 \%$. IR (KBr): 3192, $3126(\mathrm{NH}), 2961,2922,2876\left(\mathrm{CH}_{2}, \mathrm{CH}_{3}\right)$, $2263(\mathrm{C} \equiv \mathrm{N}), 1681(\mathrm{C}=\mathrm{O}), 1646,1618(\mathrm{C}=\mathrm{N}) \mathrm{cm}^{-1 .}{ }^{1} \mathrm{H}$ NMR (400 MHz, DMSO-d $\left.{ }_{6}\right) \delta(\mathrm{ppm}): 7.57-7.47(\mathrm{~m}, 5 \mathrm{H}$, $\mathrm{Ar}-\mathrm{H})$, for anti-isomer [11.69 (s, 1H, NH, exchangeable with $\left.\mathrm{D}_{2} \mathrm{O}\right), 7.96(\mathrm{~s}, 1 \mathrm{H}, \mathrm{HC}=\mathrm{N}), 4.12\left(\mathrm{~s}, 2 \mathrm{H}, \mathrm{CH}_{2} \mathrm{CN}\right)$, $\left.2.41\left(\mathrm{~s}, 3 \mathrm{H}, \mathrm{CH}_{3}\right)\right]$, for syn-isomer $[11.63(\mathrm{~s}, 1 \mathrm{H}, \mathrm{NH}$, exchangeable with $\left.\mathrm{D}_{2} \mathrm{O}\right), 8.13(\mathrm{~s}, 1 \mathrm{H}, \mathrm{HC}=\mathrm{N}), 3.76(\mathrm{~s}$, $\left.\left.2 \mathrm{H}, \mathrm{CH}_{2} \mathrm{CN}\right), 2.04\left(\mathrm{~s}, 3 \mathrm{H}, \mathrm{CH}_{3}\right)\right]$. $\mathrm{MS} \mathrm{m} / \mathrm{z}(\%): 301\left(\mathrm{M}^{+}\right.$; 10.4), 265 (11.7), 141 (5.0), 128 (33.8), 118 (19.0), 77 (100), 51 (24.2). Anal. Calcd. for $\mathrm{C}_{14} \mathrm{H}_{12} \mathrm{ClN}_{5} \mathrm{O}$ (301.73): C 55.73; H 4.01; Cl 11.75; N 23.21. Found: C 55.71; H $3.99 ; \mathrm{Cl} 11.74 ; \mathrm{N} 23.18$.

\section{1,2-Bis((5-chloro-3-methyl-1-phenyl-1H-pyrazol-4-yl)meth- ylene)hydrazine 4}

Method A A mixture of 5-chloro-3-methyl-1-phenyl$1 H$-pyrazole-4-carbaldehyde $1(2.2 \mathrm{~g}, 0.01 \mathrm{~mol})$ and cyanoacetohydrazide $2(0.99 \mathrm{~g}, 0.01 \mathrm{~mol})$ in ethanol $(30 \mathrm{ml})$ containing drops of piperidine was heated under reflux for $2 \mathrm{~h}$. The light brown solid which separated after cooling was collected by filtration and then recrystallized from ethanol to give 4 , yield: $79 \%$.

Method B Compound $3(0.5 \mathrm{~g}, 1.7 \mathrm{mmol})$ was heated under reflux in pyridine $(10 \mathrm{ml})$ for $4 \mathrm{~h}$. The light brown solid which separated after cooling was collected by filtration and then recrystallized from ethanol to give 4 , yield: $44 \%$.

Method C A mixture of compound $3(0.5 \mathrm{~g}, 1.7 \mathrm{mmol})$ and anhydrous potassium carbonate $(1.38 \mathrm{~g}, 0.01 \mathrm{~mol})$ in dry acetone $(30 \mathrm{ml})$ was heated under reflux in water bath for $24 \mathrm{~h}$. After cooling, poured onto cooled water to afford light 
brown solid that was recrystallized from ethanol to give $\mathbf{4}$, yield: $41 \%$.

Method D A mixture of $\mathbf{3}(1.0 \mathrm{~g}, 3.3 \mathrm{mmol})$ and hydrazine monohydrate $(0.16 \mathrm{ml} 3.3 \mathrm{mmol})$ in dioxane $(20 \mathrm{ml})$ was heated under reflux for $3 \mathrm{~h}$. After cooling, the solid deposited was collected by filtration and recrystallized from ethanol to give 4 , yield: $67 \%$.

Method E A mixture of $9(1.0 \mathrm{~g}, 2.3 \mathrm{mmol})$ and hydrazine monohydrate $(0.12 \mathrm{ml}, 2.3 \mathrm{mmol})$ in ethanol $(20 \mathrm{ml})$ was heated under reflux for $3 \mathrm{~h}$. After slow evaporation, pour onto acidified cold water, the solid deposited was collected by filtration and recrystallized from ethanol to give $\mathbf{4}$, yield: $64 \%$.

Method F A mixture of $\mathbf{2 3}(1.0 \mathrm{~g}, 2.2 \mathrm{mmol})$ and hydrazine monohydrate $(0.11 \mathrm{ml}, 2.2 \mathrm{mmol})$ in ethanol $(20 \mathrm{ml})$ was heated under reflux for $3 \mathrm{~h}$. After slow evaporation, the solid deposited was recrystallized from ethanol to give $\mathbf{4}$, yield: $61 \%$.

4: As buff crystals; m.p.: $250{ }^{\circ} \mathrm{C}$. IR (KBr): 2998,2932 $\left(\mathrm{CH}_{3}\right), 1632(\mathrm{C}=\mathrm{N}) \mathrm{cm}^{-1 .}{ }^{1} \mathrm{H}$ NMR $\left(400 \mathrm{MHz}\right.$, DMSO-d $\left.\mathrm{d}_{6}\right)$ $\delta$ (ppm): $8.57(\mathrm{~s}, 2 \mathrm{H}, \mathrm{HC}=\mathrm{N}), 7.62-7.51(\mathrm{~m}, 10 \mathrm{H}, \mathrm{Ar}-\mathrm{H})$, $2.51\left(\mathrm{~s}, 6 \mathrm{H}, 2 \mathrm{CH}_{3}\right) . \mathrm{MS} \mathrm{m} / \mathrm{z}(\%): 217\left(\mathrm{M}^{+}-\mathrm{C}_{11} \mathrm{H}_{9} \mathrm{ClN}_{3}\right.$; 100), 182 (62.4), 156 (52.1), 141 (33.7), 105 (11.9) 77 (55.9), 55 (23.8). Anal. Calcd. for $\mathrm{C}_{22} \mathrm{H}_{18} \mathrm{Cl}_{2} \mathrm{~N}_{6}$ (437.33): $\mathrm{C}$ 60.42; H 4.15; Cl 16.21; N 19.22. Found: C 60.40; H 4.16; Cl 16.23; N 19.19 .

5'-Chloro-3'-methyl-5-oxo-1'-phenyl-2,5-dihydro-1H, 1'H-[3, 4'-bipyrazole]-4-carbonitrile 5 and 5'-chloro-3'-methyl-5-0 xo-1'-phenyl-4,5-dihydro-1H,1'H-[3,4'-bipyrazole]-4-carbox amide 6 (as lactam-lacim tautomer)

Method A Compound $1(0.5 \mathrm{~g}, 2.3 \mathrm{mmol})$ and cyanoacetohydrazide $2(0.23 \mathrm{~g}, 2.3 \mathrm{mmol})$ are mixed together in absolute ethanol $(15 \mathrm{ml})$ containing few drops of glacial acetic acid $(0.5 \mathrm{ml})$. The reaction mixture was refluxed for $10 \mathrm{~h}$ and left to cool, the solid formed was filtered and then recrystallized from ethanol to give $\mathbf{5}$, yield: $\mathbf{7 3 \%}$.

Method B Compound 3 (0.5 g, $1.7 \mathrm{mmol})$ was heated under reflux in pyridine $(10 \mathrm{ml})$ for $4 \mathrm{~h}$. The light brown solid which separated after cooling was collected by filtration and then recrystallized from ethanol to give 4 . After acidification of the mother liquor, the solid deposited was filtrate and then recrystallized from ethanol to give $\mathbf{5}$, yield: $31 \%$.

Method C Compound 3 (0.5 g, $1.7 \mathrm{mmol})$ was heated under reflux in glacial acetic acid $(10 \mathrm{ml})$ for $10 \mathrm{~h}$. The reaction mixture was then partially evaporated and left to precipitate, the product obtained was filtered and then recrystallized from benzene to give $\mathbf{6}$, yield $33 \%$, while the insoluble fractional in benzene was recrystallized from ethanol to afford $\mathbf{5}$, yield: $46 \%$.

5: As brown crystals; m.p.: $>360{ }^{\circ} \mathrm{C}$. IR (KBr): 3374 , $3217(\mathrm{NH}), 2927,2857\left(\mathrm{CH}_{3}\right), 2213(\mathrm{C} \equiv \mathrm{N}), 1672(\mathrm{C}=\mathrm{O})$ $\mathrm{cm}^{-1.1}{ }^{\mathrm{H}}$ NMR (400 MHz, DMSO-d $\left.{ }_{6}\right) \delta$ (ppm): 11.82 (br.s, $1 \mathrm{H}, \mathrm{NH}$, exchangeable with $\mathrm{D}_{2} \mathrm{O}$ ), 11.19 (br.s, $1 \mathrm{H}, \mathrm{NH}$, exchangeable with $\left.\mathrm{D}_{2} \mathrm{O}\right), 7.81-7.24(\mathrm{~m}, 5 \mathrm{H}, \mathrm{Ar}-\mathrm{H}), 2.20$ (s, 3H, $\mathrm{CH}_{3}$ ). MS m/z (\%): $299\left(\mathrm{M}^{+} ; 16.1\right), 217$ (13.2), 188 (100), 182 (9.0), 174 (35.9), 105 (25.7), 91 (33.8), 77 (64.9). Anal. Calcd. for $\mathrm{C}_{14} \mathrm{H}_{10} \mathrm{ClN}_{5} \mathrm{O}$ (299.72): C 56.10; $\mathrm{H}$ 3.36; Cl 11.83; N 23.37. Found: C 56.08; H 3.34; Cl 11.86; N 23.39.

6 (As lactam-lactim tautomer): as orange crystals; m.p.: $210-211^{\circ} \mathrm{C}$. IR (KBr): 3418, 3294, $3120\left(\mathrm{NH}_{2}\right.$, $\mathrm{NH}), 2926,2850\left(\mathrm{CH}, \mathrm{CH}_{3}\right), 1687,1641(\mathrm{C}=\mathrm{O}) \mathrm{cm}^{-1 .}{ }^{1} \mathrm{H}$ NMR (400 MHz, DMSO-d $\left.{ }_{6}\right) \delta(\mathrm{ppm}): 13.31(\mathrm{~s}, 1 \mathrm{H}, \mathrm{OH}$, exchangeable with $\mathrm{D}_{2} \mathrm{O}$ for lactim tautomer), 12.20 (s, $2 \mathrm{H}, \mathrm{NH}_{2}$, exchangeable with $\mathrm{D}_{2} \mathrm{O}$ for lactam tautomer), $10.65\left(\mathrm{~s}, 1 \mathrm{H}, \mathrm{NH}\right.$, exchangeable with $\mathrm{D}_{2} \mathrm{O}$ for lactam tautomer), $10.40\left(\mathrm{~s}, 2 \mathrm{H}, \mathrm{NH}_{2}\right.$, exchangeable with $\mathrm{D}_{2} \mathrm{O}$ for lactim tautomer), $7.69(\mathrm{~d}, 2 \mathrm{H}, \mathrm{Ar}-\mathrm{H}, J=8.8 \mathrm{~Hz}), 7.50$ (t, $2 \mathrm{H}, \mathrm{Ar}-\mathrm{H}, J=7.6, J=8.4 \mathrm{~Hz}), 7.34-7.28(\mathrm{~m}, 1 \mathrm{H}, \mathrm{Ar}-\mathrm{H})$, $3.46(\mathrm{~s}, 1 \mathrm{H}, \mathrm{CHCO}), 2.15,2.05\left(2 \mathrm{~s}, 3 \mathrm{H}, \mathrm{CH}_{3}\right) . \mathrm{MS} \mathrm{m} / \mathrm{z}$ (\%): $317\left(\mathrm{M}^{+} ; 15.0\right), 316$ (71.3), 299 (25.0), 281 (100), 274 (8.1), 265 (27.3), 77 (91.1), 51 (30.2). Anal. Calcd. for $\mathrm{C}_{14} \mathrm{H}_{12} \mathrm{ClN}_{5} \mathrm{O}_{2}$ (317.73): C 52.92; $\mathrm{H} 3.81 ; \mathrm{Cl} \mathrm{11.16;} \mathrm{N}$ 22.04. Found: C 52.92; H 3.83; Cl 11.13; N 22.01.

\section{$N^{\prime}$-((5-Chloro-3-methyl-1-phenyl-1H-pyrazol-4-yl) methylene)-2-cyano-3-(4-nitrophenyl)acrylohydrazide 9 (as lactam-lacim tautomer)}

A mixture of 3 (1.0 g, $3.3 \mathrm{mmol})$, p-nitrobenzaldehyde $(0.50 \mathrm{~g}, 3.3 \mathrm{mmol})$ and sodium ethoxide $(15 \mathrm{ml})$ in absolute ethanol $(20 \mathrm{ml})$ was stirred at ambient temperature for $4 \mathrm{~h}$. The resulting mixture was acidified with cold dilute acetic acid. The precipitated solid was filtered off, washed several times with cold water, and recrystallized from ethanol to give 9 as yellow crystals; m.p.: $216-218{ }^{\circ} \mathrm{C}$, yield: $71 \%$. IR (KBr): 3273, $3194(\mathrm{NH}), 2962,2923,2854\left(\mathrm{CH}_{3}\right), 2210$ $(\mathrm{C} \equiv \mathrm{N}), 1680,1668(\mathrm{C}=\mathrm{O}), 1626(\mathrm{C}=\mathrm{N}), 1604(\mathrm{C}=\mathrm{C}), 1529$, $1346\left(\mathrm{NO}_{2}\right) \mathrm{cm}^{-1.1} \mathrm{HNMR}$ (400 MHz, DMSO-d6) $\delta$ (ppm): $11.60\left(\mathrm{~s}, 1 \mathrm{H}, \mathrm{OH}\right.$, exchangeable with $\mathrm{D}_{2} \mathrm{O}$ for lactim tautomer), 8.57 (s, $1 \mathrm{H}, \mathrm{NH}$, exchangeable with $\mathrm{D}_{2} \mathrm{O}$ for lactam tautomer), $8.47(\mathrm{~s}, 1 \mathrm{H}, \mathrm{HC}=\mathrm{N}), 8.24-8.22(\mathrm{~d}, 2 \mathrm{H}, \mathrm{Ar}-\mathrm{H}$, $J=8.4 \mathrm{~Hz}), 8.01$ (s, 1H, HC (olefinic) $), 7.73-7.71$ (d, 2H, Ar-H, $J=8.4 \mathrm{~Hz}$ ), 7.59-7.49 (m, 5H, $\mathrm{Ar}-\mathrm{H}), 2.51\left(\mathrm{~s}, 3 \mathrm{H}, \mathrm{CH}_{3}\right)$. MS m/z (\%): 434 (M. $\left.^{+} ; 4.4\right), 342$ (100), 324 (16.0), 316 (27.5), 299 (7.7), 281 (5.4), 265 (10.5), 77 (57.9). Anal. Calcd. for $\mathrm{C}_{21} \mathrm{H}_{15} \mathrm{ClN}_{6} \mathrm{O}_{3}$ (434.84): C 58.01; H 3.48; Cl 8.15; N 19.33. Found: C 57.97; H 3.46; Cl 8.13; N 19.36. 
$N^{\prime}$-((5-Chloro-3-methyl-1-phenyl-1H-pyrazol-4-yl) methylene)-2-cyano-2-(2-oxoindolin-3-ylidene)acetohydrazide 11

A mixture of $3(1.0 \mathrm{~g}, 3.3 \mathrm{mmol})$, isatin $(0.49 \mathrm{~g}, 3.3 \mathrm{mmol})$, and piperidine $(0.5 \mathrm{ml})$ in absolute ethanol $(20 \mathrm{ml})$ was heated under reflux for $4 \mathrm{~h}$. After cooling, the solid deposited was collected by filtration, and recrystallized from ethanol to give $\mathbf{1 1}$ as dark brown crystals; m.p.: $284-285{ }^{\circ} \mathrm{C}$, yield: 53\%. IR (KBr): 3273, $3194(\mathrm{NH}), 2961,2923,2876$ $\left(\mathrm{CH}_{3}\right), 2202(\mathrm{C} \equiv \mathrm{N}), 1728,1680(\mathrm{C}=\mathrm{O}), 1621(\mathrm{C}=\mathrm{N}), 1604$ $(\mathrm{C}=\mathrm{C}) \mathrm{cm}^{-1.1} \mathrm{H}$ NMR $\left(400 \mathrm{MHz}, \mathrm{DMSO}-\mathrm{d}_{6}\right) \delta(\mathrm{ppm}): 12.35$ (br.s, $1 \mathrm{H}, \mathrm{NH}$, exchangeable with $\mathrm{D}_{2} \mathrm{O}$ ), 11.26 (br.s, $1 \mathrm{H}$, $\mathrm{NH}$, exchangeable with $\left.\mathrm{D}_{2} \mathrm{O}\right), 8.55\left(\mathrm{~d}, 1 \mathrm{H}, \mathrm{Ar}-\mathrm{H}_{\text {indolinone, }}\right.$, $J=10 \mathrm{~Hz}), 8.52(\mathrm{~s}, 1 \mathrm{H}, \mathrm{HC}=\mathrm{N}), 7.67-7.49(\mathrm{~m}, 5 \mathrm{H}, \mathrm{Ar}-\mathrm{H})$, $7.43\left(\mathrm{~d}, \mathrm{~d}, 1 \mathrm{H}, \mathrm{Ar}-\mathrm{H}_{\text {indolinone }}, J=8.4 \mathrm{~Hz}\right), 7.31(\mathrm{t}, 1 \mathrm{H}$, Ar- $\left.\mathrm{H}_{\text {indolinone }}, J=7.6, J=7.2 \mathrm{~Hz}\right), 6.88\left(\mathrm{~d}, 1 \mathrm{H}, \mathrm{Ar}-\mathrm{H}_{\text {indolinone, }}\right.$, $J=8 \mathrm{~Hz}), 2.60\left(\mathrm{~s}, 3 \mathrm{H}, \mathrm{CH}_{3}\right)$. MS m/z (\%): $431\left(\mathrm{M}^{++}+1\right.$; 13.6), 395 (25.6), 217 (100), 183 (19.7), 77 (57.9). Anal. Calcd. for $\mathrm{C}_{22} \mathrm{H}_{15} \mathrm{ClN}_{6} \mathrm{O}_{2}$ (430.85): C 61.33; $\mathrm{H}$ 3.51; $\mathrm{Cl}$ 8.23; N 19.51. Found: C 61.35; H 3.49; Cl 8.21; N 19.53.

1-(((5-Chloro-3-methyl-1-phenyl-1H-pyrazol-4-yl)methylene)amino)-4,6-dimethyl-2-oxo-1,2-dihydropyridine-3-carbonitrile 12

A mixture of $\mathbf{3}(0.3 \mathrm{~g}, 1 \mathrm{mmol})$ and acetylacetone $(0.10 \mathrm{ml}$, $1 \mathrm{mmol})$ in ethanol $(15 \mathrm{ml})$ containing a few drops of piperidine (four drops) was refluxed for $6 \mathrm{~h}$. After cooling, the reaction mixture was acidified with cold dilute acetic acid. The solid that separated out was filtered off, washed several times with cold water and recrystallized from ethanol to give 12 as buff crystals; m.p.: $243-245{ }^{\circ} \mathrm{C}$, yield: $55 \%$. IR (KBr): 2999, 2928, $2849\left(\mathrm{CH}_{3}\right), 2215(\mathrm{C} \equiv \mathrm{N}), 1651$ $(\mathrm{C}=\mathrm{O}), 1591(\mathrm{C}=\mathrm{N}) \mathrm{cm}^{-1 .}{ }_{1} \mathrm{H}$ NMR $\left(400 \mathrm{MHz}, \mathrm{DMSO}_{6}\right)$ $\delta(\mathrm{ppm}): 8.76(\mathrm{~s}, 1 \mathrm{H}, \mathrm{HC}=\mathrm{N}), 7.62-7.51(\mathrm{~m}, 5 \mathrm{H}, \mathrm{Ar}-\mathrm{H})$, 6.39 (s, $1 \mathrm{H}, \mathrm{CH}_{\text {pyridinone }}$ ), 2.47 (s, 3H, $\mathrm{CH}_{\text {3pyrazole }}$ ), 2.346 (s, $\left.3 \mathrm{H}, \mathrm{CH}_{3 \text { pyridinone }}\right), 2.343$ (s, 3H, $\left.\mathrm{CH}_{3 \text { pyridinone }}\right)$. $\mathrm{MS} \mathrm{m} / \mathrm{z}(\%)$ : 365 ( $\left.\mathrm{M}^{+} ; 5.8\right), 218$ (51.2), 183 (27.3), 156 (16.8), 77 (100), 51 (28.6). Anal. Calcd. for $\mathrm{C}_{19} \mathrm{H}_{16} \mathrm{ClN}_{5} \mathrm{O}$ (365.82): $\mathrm{C} 62.38$; H 4.41; Cl 9.69; N 19.14. Found: C 62.35; H 4.43; Cl 9.66; N 19.10 .

6-Amino-1-(((5-chloro-3-methyl-1-phenyl-1H-pyrazol-4-yl) methylene)amino)-4-(3,4-dimethoxyphenyl)-2-oxo-1,2,3,4 -tetrahydropyridine-3,5-dicarbonitrile 13

A mixture of 3 (0.3 g, $1 \mathrm{mmol})$, 2-(3,4-dimethoxybenzylidene)malononitrile $(0.21 \mathrm{~g}, 1 \mathrm{mmol})$ in absolute ethanol $(15 \mathrm{ml})$ containing few drops of piperidine (four drops) was heated under reflux for $6 \mathrm{~h}$. After evaporation of the solvent in vacuo, the solid obtained was collected and recrystallized from benzene to give $\mathbf{1 3}$ as reddish brown crystals; m.p.: $192-194{ }^{\circ} \mathrm{C}$, yield: $61 \%$. IR ( $\left.\mathrm{KBr}\right): 3341,3216\left(\mathrm{NH}_{2}\right)$, 2935, $2837\left(\mathrm{CH}_{3}\right), 2212(\mathrm{C} \equiv \mathrm{N}), 1676(\mathrm{C}=\mathrm{O}), 1628(\mathrm{C}=\mathrm{N})$, $1606(\mathrm{C}=\mathrm{C}) \mathrm{cm}^{-1 .}{ }^{1} \mathrm{H}$ NMR $\left(400 \mathrm{MHz}, \mathrm{DMSO}_{6}\right) \delta(\mathrm{ppm})$ : 8.25 (br.s, $2 \mathrm{H}, \mathrm{NH}_{2}$, exchangeable with $\left.\mathrm{D}_{2} \mathrm{O}\right), 7.79$ (s, $1 \mathrm{H}$, $\mathrm{HC}=\mathrm{N}), 7.65-7.50(\mathrm{~m}, 5 \mathrm{H}, \mathrm{Ar}-\mathrm{H}), 7.34(\mathrm{~s}, 1 \mathrm{H}, \mathrm{Ar}-\mathrm{H})$, 7.08-7.06 (d, 2H, Ar-H), 3.85 (s, 3H, $\left.\mathrm{OCH}_{3}\right), 3.82$ (s, 3H, $\left.\mathrm{OCH}_{3}\right), 3.79-3.77$ (d, $\left.1 \mathrm{H}, \mathrm{COCHCN}, J=6.8 \mathrm{~Hz}\right), 3.69-3.67$ (d, $1 \mathrm{H}, \mathrm{CHAr}, J=8 \mathrm{~Hz}$ ), 2.27 (s, 3H, $\mathrm{CH}_{3}$ ). MS m/z (\%): 343 $\left(\mathrm{M}^{+}-\left[\mathrm{C}_{6} \mathrm{H}_{3}(\mathrm{OMe})_{2}+\mathrm{Cl}\right] ; 17.1\right), 341$ (100), 327 (15.3), 301 (3.4), 77 (28.8). Anal. Calcd. for $\mathrm{C}_{26} \mathrm{H}_{22} \mathrm{ClN}_{7} \mathrm{O}_{3}$ (515.96): C 60.53; H 4.30; Cl 6.87; N 19.00. Found: C 60.55; H 4.31; $\mathrm{Cl} 6.85 ; \mathrm{N} 18.98$.

\section{$N^{\prime}$-((5-Chloro-3-methyl-1-phenyl-1H-pyrazol-4-yl) methylene)-2-imino-2H-chromene-3-carbohydrazide 15a}

A mixture of 3 (1.0 g, $3.3 \mathrm{mmol})$ and salicylaldehyde 14a $(0.35 \mathrm{ml}, 3.3 \mathrm{mmol})$ in absolute ethanol $(20 \mathrm{ml})$ in the presence of piperidine $(0.5 \mathrm{ml})$ was heated under reflux for $3 \mathrm{~h}$. After cooling, the solid deposited was collected by filtration and recrystallized from ethanol to give $\mathbf{1 5 a}$ as light yellow crystals; m.p.: $210-211^{\circ} \mathrm{C}$, yield: $85 \%$. IR $(\mathrm{KBr}): 3312(\mathrm{NH}), 2958,2921,2854\left(\mathrm{CH}_{3}\right), 1682(\mathrm{C}=\mathrm{O})$, $1632(\mathrm{C}=\mathrm{N}), 1617(\mathrm{C}=\mathrm{C}) \mathrm{cm}^{-1 .}{ }^{1} \mathrm{H}$ NMR $(400 \mathrm{MHz}$, DMSO- $\left.\mathrm{d}_{6}\right) \delta(\mathrm{ppm}): 13.46(\mathrm{~s}, 1 \mathrm{H}, \mathrm{NH}$, exchangeable with $\left.\mathrm{D}_{2} \mathrm{O}\right), 9.17\left(\mathrm{~s}, 1 \mathrm{H}, \mathrm{NH}\right.$, exchangeable with $\left.\mathrm{D}_{2} \mathrm{O}\right), 8.49$ $(\mathrm{s}, 1 \mathrm{H}, \mathrm{HC}=\mathrm{C}), 8.29(\mathrm{~s}, 1 \mathrm{H}, \mathrm{HC}=\mathrm{N}), 7.81(\mathrm{~d}, 1 \mathrm{H}, \mathrm{Ar}-\mathrm{H}$, $J=7.6 \mathrm{~Hz}), 7.59-7.49(\mathrm{~m}, 7 \mathrm{H}, \mathrm{Ar}-\mathrm{H}), 7.29-7.23(\mathrm{~d}, \mathrm{~d}, 1 \mathrm{H}$, $\mathrm{Ar}-\mathrm{H}, J=7.6, J=8.4 \mathrm{~Hz}), 2.65\left(\mathrm{~s}, 3 \mathrm{H}, \mathrm{CH}_{3}\right) . \mathrm{MS} \mathrm{m} / \mathrm{z}$ (\%): 339 (M. $\left.{ }^{+}-\mathrm{C}_{4} \mathrm{H}_{2} \mathrm{O} ; 23.6\right), 338$ (100), 321 (32.6), 303 (44.9), 261 (2.8), 218 (14.7), 217 (22.0), 182 (15.0), 155 (9.6), 128 (14.9), 77 (46.9).Anal. Calcd. for $\mathrm{C}_{21} \mathrm{H}_{16} \mathrm{ClN}_{5} \mathrm{O}_{2}$ (405.84): C 62.15; H 3.97; Cl 8.73; N 17.26. Found: C $62.19 ; \mathrm{H} 3.98 ; \mathrm{Cl} 8.71 ; \mathrm{N} 17.24$.

\section{$N^{\prime}$-((5-Chloro-3-methyl-1-phenyl-1H-pyrazol-4-yl) methylene)-2-imino-6,8-dinitro-2H-chromene-3-carbohy- drazide 15b}

A mixture of $3(1.0 \mathrm{~g}, 3.3 \mathrm{mmol})$ and 3,5-dinitrosalicylaldehyde 14b $(0.7 \mathrm{~g}, 3.3 \mathrm{mmol})$ in absolute ethanol $(20 \mathrm{ml})$ in the presence of piperidine $(0.5 \mathrm{ml})$ was heated under reflux for $3 \mathrm{~h}$. After cooling, the solid deposited was collected by filtration and recrystallized from dioxane to give $15 b$ as yellow crystals; m.p.: $248{ }^{\circ} \mathrm{C}$, yield: $88 \%$. IR (KBr): $3206(\mathrm{NH}), 2962,2926,2863\left(\mathrm{CH}_{3}\right), 1683(\mathrm{C}=\mathrm{O})$, $1619(\mathrm{C}=\mathrm{N}), 1550,1339\left(\mathrm{NO}_{2}\right) \mathrm{cm}^{-1 .}{ }^{1} \mathrm{H}$ NMR $(400 \mathrm{MHz}$, DMSO-d $\left.{ }_{6}\right) \delta(\mathrm{ppm}): 11.81(\mathrm{~s}, 1 \mathrm{H}, \mathrm{NH}$, exchangeable with $\left.\mathrm{D}_{2} \mathrm{O}\right), 8.98\left(\mathrm{~s}, 1 \mathrm{H}, \mathrm{NH}\right.$, exchangeable with $\left.\mathrm{D}_{2} \mathrm{O}\right)$, $8.50(\mathrm{~s}, 1 \mathrm{H}, \mathrm{Ar}-\mathrm{H}), 8.30(\mathrm{~s}, 1 \mathrm{H}, \mathrm{HC}=\mathrm{C}), 8.19(\mathrm{~s}, 1 \mathrm{H}$, $\mathrm{HC}=\mathrm{N}), 8.13(\mathrm{~s}, 1 \mathrm{H}, \mathrm{Ar}-\mathrm{H}), 7.59-7.47(\mathrm{~m}, 5 \mathrm{H}, \mathrm{Ar}-\mathrm{H})$, 2.45(s, 3H, $\left.\mathrm{CH}_{3}\right) . \mathrm{MS} \mathrm{m} / \mathrm{z}(\%): 219\left(\mathrm{M}^{+}{ }^{+}-\mathrm{C}_{10} \mathrm{H}_{4} \mathrm{~N}_{4} \mathrm{O}_{6}\right.$; 
100), 156 (13.3), 77 (25.6), 51 (12.6). Anal. Calcd. for

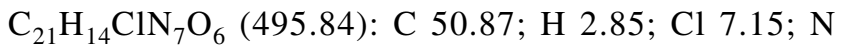
19.77. Found: C 50.89; H 2.82; Cl 7.17; N 19.79.

\section{$\mathrm{N}$-(3-(2-((5-Chloro-3-methyl-1-phenyl-1H-pyrazol-4-yl)} methylene)hydrazine-1-carbonyl)-2H-chromen-2-ylidene) formamide 16

A solution of compound 15a $(0.25 \mathrm{~g}, 0.62 \mathrm{mmol})$ in a mixture of triethylorthoformate $(5 \mathrm{ml})$ and acetic anhydride $(5 \mathrm{ml})$ was heated under reflux for $3 \mathrm{~h}$. After cooling, the yellow precipitated was collected by filtration and recrystallized from toluene to give $\mathbf{1 6}$ as yellow crystals; m.p.: $241-243{ }^{\circ} \mathrm{C}$, yield: $65 \%$. IR (KBr): $3209(\mathrm{NH}), 2958$, 2923, $2854\left(\mathrm{CH}_{3}\right), 1710\left(\mathrm{C}=\mathrm{O}_{\text {aldehyde }}\right), 1677\left(\mathrm{C}=\mathrm{O}_{\text {amide }}\right)$, $1616(\mathrm{C}=\mathrm{N}) \mathrm{cm}^{-1 .}{ }^{1} \mathrm{H}$ NMR $\left(400 \mathrm{MHz}, \mathrm{DMSO}-\mathrm{d}_{6}\right) \delta$ (ppm): 11.69 (s, 1H, NH, exchangeable with $\left.\mathrm{D}_{2} \mathrm{O}\right), 8.83$ (s, $1 \mathrm{H}, \mathrm{CHO}), 8.43(\mathrm{~s}, 1 \mathrm{H}, \mathrm{HC}=\mathrm{C}), 8.27(\mathrm{~s}, 1 \mathrm{H}, \mathrm{HC}=\mathrm{N})$, $7.98(\mathrm{~d}, 1 \mathrm{H}, \mathrm{Ar}-\mathrm{H}, J=7.6 \mathrm{~Hz}), 7.80-7.74(\mathrm{~d}, \mathrm{~d}, 1 \mathrm{H}, \mathrm{Ar}-\mathrm{H}$, $J=8.4, J=8.4 \mathrm{~Hz}) 7.60-7.50(\mathrm{~m}, 5 \mathrm{H}, \mathrm{Ar}-\mathrm{H}), 7.47-7.45$ $(\mathrm{d}, 1 \mathrm{H}, \mathrm{Ar}-\mathrm{H}, J=7.6 \mathrm{~Hz}), 7.43-7.37$ (d,d, 1H, Ar-H, $J=8.4, J=7.6 \mathrm{~Hz}), 2.43\left(\mathrm{~s}, 3 \mathrm{H}, \mathrm{CH}_{3}\right)$. MS m/z (\%): 432 (M. ${ }^{+}-1$; 11.7), 362 (27.1), 217 (100), 77 (54.3). Anal. Calcd. for $\mathrm{C}_{22} \mathrm{H}_{16} \mathrm{ClN}_{5} \mathrm{O}_{3}$ (433.85): C 60.91; $\mathrm{H} 3.72 ; \mathrm{Cl}$ 8.17; N 16.14. Found: C 60.93; H 3.69; Cl 8.14; N 16.17.

$\mathrm{N}$-(3-(2-((5-Chloro-3-methyl-1-phenyl-1H-pyrazol-4-yl)methylene)hydrazine-1-carbonyl)-6,8-dinitro-2H-chromen-2-ylidene)formamide $17 \mathrm{a}$ and 3-(((5-chloro-3-methyl-1-phenyl-1H-pyrazol-4-yl) methylene)amino)-2-ethoxy-7,9-dinitro-2,3-dihydro-4H-chromeno[2,3-d]pyrimidin-4-one 17b

A solution of compound $\mathbf{1 5 b}(0.25 \mathrm{~g}, 0.51 \mathrm{mmol})$ in a mixture of triethylorthoformate $(5 \mathrm{ml})$ and acetic anhydride $(5 \mathrm{ml})$ was heated under reflux for $3 \mathrm{~h}$. After cooling, the solid deposited was collected by filtration and recrystallized from toluene to give 17a,b as yellow crystals; m.p.: 203-205 ${ }^{\circ} \mathrm{C}$, yield: $67 \%$.

IR (KBr): $3443(\mathrm{NH}), 2973,2927,2861\left(\mathrm{C}-\mathrm{H}_{\text {aliph. }}\right), 1719$ $\left(\mathrm{C}=\mathrm{O}_{\text {aldehyde }}\right), 1678\left(\mathrm{C}=\mathrm{O}_{\text {amide }}\right), 1631(\mathrm{C}=\mathrm{N}), 1613(\mathrm{C}=\mathrm{C})$, 1528, $1346\left(\mathrm{NO}_{2}\right) \mathrm{cm}^{-1.1} \mathrm{H}$ NMR (400 MHz, DMSO-d $\left.{ }_{6}\right) \delta$ (ppm):

17a: 9.03 (s, 1H, CHO), 8.79 (s, 1H, Ar-H), 8.72 (s, 1H, $\mathrm{NH}$, exchangeable with $\left.\mathrm{D}_{2} \mathrm{O}\right), 8.59(\mathrm{~s}, 1 \mathrm{H}, \mathrm{HC}=\mathrm{C}), 8.48(\mathrm{~s}$, $1 \mathrm{H}, \mathrm{HC}=\mathrm{N}), 8.32(\mathrm{~s}, 1 \mathrm{H}, \mathrm{Ar}-\mathrm{H}), 7.67-7.52(\mathrm{~m}, 5 \mathrm{H}, \mathrm{Ar}-\mathrm{H})$, 2.51(s, $\left.3 \mathrm{H}, \mathrm{CH}_{3}\right)$.

17b: $8.69(\mathrm{~s}, 1 \mathrm{H}, \mathrm{Ar}-\mathrm{H}), 8.58(\mathrm{~s}, 1 \mathrm{H}, \mathrm{HC}=\mathrm{C}), 8.41(\mathrm{~s}$, $1 \mathrm{H}, \mathrm{HC}=\mathrm{N}), 8.30(\mathrm{~s}, 1 \mathrm{H}, \mathrm{Ar}-\mathrm{H}), 7.67-7.52(\mathrm{~m}, 5 \mathrm{H}, \mathrm{Ar}-\mathrm{H})$, $5.83\left(\mathrm{~s}, 1 \mathrm{H}, \mathrm{CH}_{\text {pyrimidinone }}\right), 3.66-3.56\left(\mathrm{q}, 2 \mathrm{H}, \mathrm{CH}_{2} \mathrm{CH}_{3}\right.$, $J=7.2 \mathrm{~Hz}), 2.50\left(\mathrm{~s}, 3 \mathrm{H}, \mathrm{CH}_{3}\right), 1.03-1.00\left(\mathrm{t}, 3 \mathrm{H}, \mathrm{CH}_{2} \mathrm{CH}_{3}\right.$, $J=6.8 \mathrm{~Hz}$ ).
GC-MS: 2 peaks; 1 st peak; percentage $=16.88 \% ; \mathrm{MS} \mathrm{m} / \mathrm{z}$ (\%): $219\left(\mathrm{M}^{+}{ }^{+} \mathrm{C}_{11} \mathrm{H}_{5} \mathrm{~N}_{4} \mathrm{O}_{7} ; 100\right), 217$ (8.2), 182 (5.4), 155 (20.7), 143 (6.2), 77 (37.0), 51 (17.6).

2nd peak; percentage $=83.12 \%$; MS m/z (\%): 219 (M. ${ }^{+}-\mathrm{C}_{13} \mathrm{H}_{9} \mathrm{~N}_{4} \mathrm{O}_{7}$; 92.8), 217 (100), 182 (100), 155 (70.5), 141 (100), 114 (29.5), 77 (100), 51 (68.3).

\section{4-Amino- $N$ '-((5-chloro-3-methyl-1-phenyl-1H-pyrazol-4-yl) methylene)-3-phenyl-2-thioxo-2,3-dihydrothiazole-5-car- bohydrazide 18}

To a solution of compound $\mathbf{3}(3.01 \mathrm{~g}, 0.01 \mathrm{~mol})$ in ethanol $(30 \mathrm{ml})$ containing triethylamine $(0.5 \mathrm{ml})$, elemental sulfur $(0.32 \mathrm{~g}, 0.01 \mathrm{~mol})$ and phenyl isothiocyanate $(1.2 \mathrm{ml}$, $0.01 \mathrm{~mol}$ ) were added. The reaction mixture was heated at $60{ }^{\circ} \mathrm{C}$ for $1 \mathrm{~h}$ with continuous stirring. The grey solid deposited, while hot was collected by filtration, washed several times with ethanol and recrystallized from toluene to give 18 as buff crystals; m.p.: $261-262{ }^{\circ} \mathrm{C}$, yield: $80 \%$. IR (KBr): 3382, 3247, $3178\left(\mathrm{NH}_{2}, \mathrm{NH}\right), 2958,2925,2857\left(\mathrm{CH}_{3}\right), 1632$ $(\mathrm{C}=\mathrm{O}), 1602(\mathrm{C}=\mathrm{N}), 1242(\mathrm{C}=\mathrm{S}) \mathrm{cm}^{-1.1} \mathrm{H}$ NMR $(400 \mathrm{MHz}$, $\left.\mathrm{CDCl}_{3}\right) \delta$ (ppm): 8.52 (br.s, $1 \mathrm{H}, \mathrm{NH}$, exchangeable with $\left.\mathrm{D}_{2} \mathrm{O}\right), 7.74(\mathrm{~s}, 1 \mathrm{H}, \mathrm{HC}=\mathrm{N}), 7.66-7.35(\mathrm{~m}, 10 \mathrm{H}, \mathrm{Ar}-\mathrm{H})$, 6.31 (br.s, $2 \mathrm{H}, \mathrm{NH}_{2}$, exchangeable with $\mathrm{D}_{2} \mathrm{O}$ ), 2.69 (s, $3 \mathrm{H}$, $\mathrm{CH}_{3}$ ). MS m/z (\%): 468 (M.+ $^{+}$; 12.6), 433 (17.6), 392 (37.4), 250 (100), 218 (46.7), 218 (47.4), 77 (33.7). Anal. Calcd. for $\mathrm{C}_{21} \mathrm{H}_{17} \mathrm{ClN}_{6} \mathrm{OS}_{2}$ (468.98): C 53.78; $\mathrm{H} 3.65 ; \mathrm{Cl} 7.56 ; \mathrm{N}$ 17.92; S 13.67. Found: C 53.80; H 3.66; Cl 7.53; N 17.91; S 13.65 .

6-(((5-Chloro-3-methyl-1-phenyl-1H-pyrazol-4-yl)methylene)amino)-3-phenyl-2-thioxo-2,3-dihydrothiazolo[4,5- $d$ ] pyrimidin-7(6H)-one 19

A solution of compound $\mathbf{1 6}(0.16 \mathrm{~g}, 0.34 \mathrm{mmol})$ in a mixture of triethylorthoformate $(3 \mathrm{ml})$ and acetic anhydride $(3 \mathrm{ml})$ was heated under reflux for $9 \mathrm{~h}$. After cooling, the solid deposited was collected by filtration and recrystallized from toluene to give $\mathbf{1 9}$ as off white crystals; m.p.: 258-259 ${ }^{\circ} \mathrm{C}$, yield: 83\%. IR (KBr): 2959, 2922, 2853 $\left(\mathrm{CH}_{3}\right), 1666\left(\mathrm{C}=\mathrm{O}_{\text {Pyrimidinone }}\right), 1592(\mathrm{C}=\mathrm{N}), 1243(\mathrm{C}=\mathrm{S})$ $\mathrm{cm}^{-1 .}{ }^{1} \mathrm{H}$ NMR (400 MHz, DMSO-d $\left.\mathrm{d}_{6}\right) \delta(\mathrm{ppm}): 9.31$ (s, $1 \mathrm{H}$, Ar- $\left.\mathrm{H}_{\text {Pyrimidinone }}\right), 8.22(\mathrm{~s}, 1 \mathrm{H}, \mathrm{HC}=\mathrm{N}), 7.65-7.35(\mathrm{~m}, 10 \mathrm{H}$, $\mathrm{Ar}-\mathrm{H}), 2.57$ (s, 3H, $\mathrm{CH}_{3}$ ). MS m/z (\%): $478\left(\mathrm{M}^{+} ; 8.4\right), 450$ (12.2), 443 (23.9), 402 (19.8), 343 (35.4), 261 (24.1), 217 (66.3), 77 (100). Anal. Calcd. for $\mathrm{C}_{22} \mathrm{H}_{15} \mathrm{ClN}_{6} \mathrm{OS}_{2}$ (478.97): C 55.17; H 3.16; Cl 7.40; N 17.55; S 13.39. Found: C 55.19; H 3.14; Cl 7.41; N 17.52; S 13.36. 


\section{N'-((5-Chloro-3-methyl-1-phenyl-1H-pyrazol-4-yl) methylene)-2-cyano-3-mercapto-3-(phenylamino)acrylo- hydrazide 21}

To a stirred solution of potassium hydroxide $(0.56 \mathrm{~g}$, $0.01 \mathrm{~mol})$ in DMF $(20 \mathrm{ml})$ compound $3(3.01 \mathrm{~g}, 0.01 \mathrm{~mol})$ was added. After stirring for $30 \mathrm{~min}$, phenyl isothiocyanate $(1.2 \mathrm{ml}, 0.01 \mathrm{~mol})$ was added to the resulting mixture. Stirring was persistent at ambient temperature for $12 \mathrm{~h}$. The reaction mixture was acidified with cold dilute $\mathrm{HCl}$. The solid product that separated was filtered, washed with water, and recrystallized from benzene to give $\mathbf{2 1}$ as buff crystals; m.p.: $136-138{ }^{\circ} \mathrm{C}$, yield: $72 \%$. IR (KBr): 3273, $3194(\mathrm{NH})$, 2958, 2925, $2853\left(\mathrm{CH}_{3}\right), 2225(\mathrm{C} \equiv \mathrm{N}), 1676(\mathrm{C}=\mathrm{O}), 1630$ $(\mathrm{C}=\mathrm{N}) \mathrm{cm}^{-1.1} \mathrm{H}$ NMR (400 MHz, DMSO-d 6 ) $\delta(\mathrm{ppm}): 12.70$ (s, 1H, NH, exchangeable with $\mathrm{D}_{2} \mathrm{O}$ ), 10.53 (br.s, $1 \mathrm{H}, \mathrm{NH}$, exchangeable with $\left.\mathrm{D}_{2} \mathrm{O}\right), 8.06(\mathrm{~s}, 1 \mathrm{H}, \mathrm{HC}=\mathrm{N}), 7.59-7.34(\mathrm{~m}$, $10 \mathrm{H}, \mathrm{Ar}-\mathrm{H}$ ), 2.43 (s, 3H, $\mathrm{CH}_{3}$ ), 1.21 (s, 1H, SH, exchangeable with $\left.\mathrm{D}_{2} \mathrm{O}\right)$. MS m/z (\%): 436 (M.+; 0.9), 401 (1.1), 359 (20.3), 357 (100), 343 (23.5), 316 (5.7), 281 (8.4), 128 (9.6), 77 (24.2), 51 (7.5). Anal. Calcd. for $\mathrm{C}_{21} \mathrm{H}_{17} \mathrm{ClN}_{6} \mathrm{OS}$ (436.92): C 57.73; H 3.92; Cl 8.11; N 19.24; S 7.34. Found: C 57.72; H 3.89; Cl 8.09; N 19.27; S 7.35.

\section{$N^{\prime}$-((5-Chloro-3-methyl-1-phenyl-1H-pyrazol-4-yl) methylene)-2-cyano-2-(5-oxo-3-phenylthiazoli- din-2-ylidene)acetohydrazide 22}

To a stirred solution of potassium hydroxide $(0.56 \mathrm{~g}$, $0.01 \mathrm{~mol})$ in DMF $(20 \mathrm{ml})$ compound $3(3.01 \mathrm{~g}, 0.01 \mathrm{~mol})$ was added. After stirring for $30 \mathrm{~min}$, phenyl isothiocyanate $(1.2 \mathrm{ml}, 0.01 \mathrm{~mol})$ was added to the resulting mixture. Stirring was persistent at ambient temperature for $12 \mathrm{~h}$, and then, chloroacetyl chloride $(0.8 \mathrm{ml}, 0.01 \mathrm{~mol})$ was added and stirring was persistent for additional $6 \mathrm{~h}$. The reaction mixture was acidified with cold dilute acetic acid. The separated solid was filtered off, washed several times with cold water, and recrystallized from toluene to give $\mathbf{2 2}$ as green crystals; m.p.: $140-142{ }^{\circ} \mathrm{C}$, yield: $79 \%$. IR (KBr): $3334(\mathrm{NH}), 2968,2924,2854\left(\mathrm{CH}_{2}, \mathrm{CH}_{3}\right), 2195(\mathrm{C} \equiv \mathrm{N})$, $1745\left(\mathrm{C}=\mathrm{O}_{\text {thiazolidinone }}\right), 1664\left(\mathrm{C}=\mathrm{O}_{\text {amide }}\right), 1618(\mathrm{C}=\mathrm{N}), 1595$ $(\mathrm{C}=\mathrm{C}) \mathrm{cm}^{-1.1} \mathrm{H}$ NMR (400 MHz, $\left.\mathrm{CDCl}_{3}\right) \delta(\mathrm{ppm}): 9.03$ (s, 1H, NH, exchangeable with $\left.\mathrm{D}_{2} \mathrm{O}\right), 7.99(\mathrm{~s}, 1 \mathrm{H}, \mathrm{HC}=\mathrm{N})$, 7.66-7.30 (m, 10H, Ar-H), 3.89 (s, 2H, CH $2 \mathrm{~S}), 2.58$ (s, $\left.3 \mathrm{H}, \mathrm{CH}_{3}\right)$. MS m/z (\%): $259\left(\mathrm{M}^{+}-\mathrm{C}_{11} \mathrm{H}_{9} \mathrm{ClN}_{3} ; 100\right), 244$ (29.4), 218 (17.9), 190 (4.7), 77 (7.9), 51 (4.9). Anal. Calcd. for $\mathrm{C}_{23} \mathrm{H}_{17} \mathrm{ClN}_{6} \mathrm{O}_{2} \mathrm{~S}$ (476.94): C 57.92; $\mathrm{H} 3.59 ; \mathrm{Cl} 7.43 ; \mathrm{N}$ 17.62; S 6.72. Found: C 57.94; H 3.57; Cl 7.39; N 17.60; S 6.74 .
$N^{\prime}$-((5-Chloro-3-methyl-1-phenyl-1H-pyrazol-4-yl) methylene)-2-cyano-3-(ethylthio)-3-(phenylamino)acrylohydrazide 23

To a stirred solution of potassium hydroxide $(0.56 \mathrm{~g}$, $0.01 \mathrm{~mol})$ in DMF $(20 \mathrm{ml})$ compound 3 (3.01 g, $0.01 \mathrm{~mol})$ was added. After stirring for $30 \mathrm{~min}$, phenyl isothiocyanate ( $1.2 \mathrm{ml}, 0.01 \mathrm{~mol}$ ) was added to the resulting mixture. Stirring was persistent at ambient temperature for $12 \mathrm{~h}$, and then, ethyl iodide $(0.62 \mathrm{ml}, 0.01 \mathrm{~mol})$ was added and stirring was persistent for additional $6 \mathrm{~h}$. The reaction mixture was poured onto ice cold water, and the resulting precipitate was filtered off, washed several times with cold water and recrystallized from benzene to give $\mathbf{2 3}$ as reddish brown crystals; m.p.: $178-180{ }^{\circ} \mathrm{C}$, yield: $71 \%$. IR (KBr): $3275(\mathrm{NH}), 2963$, 2925, $2858\left(\mathrm{CH}_{2}, \mathrm{CH}_{3}\right), 2193(\mathrm{C} \equiv \mathrm{N}), 1637(\mathrm{C}=\mathrm{O}), 1621$ $(\mathrm{C}=\mathrm{N}), 1590(\mathrm{C}=\mathrm{C}) \mathrm{cm}^{-1}{ }^{1} \mathrm{H} \mathrm{NMR}\left(400 \mathrm{MHz}, \mathrm{CDCl}_{3}\right) \delta$ (ppm): 12.54 (s, 1H, NH, exchangeable with $\left.\mathrm{D}_{2} \mathrm{O}\right), 9.04$ $\left(\mathrm{s}, 1 \mathrm{H}, \mathrm{NH}\right.$, exchangeable with $\left.\mathrm{D}_{2} \mathrm{O}\right), 8.03(\mathrm{~s}, 1 \mathrm{H}, \mathrm{HC}=\mathrm{N})$, 7.54-7.28 (m, 10H, Ar-H), 2.65-2.60 (q, 2H, $\underline{\mathrm{CH}}_{2} \mathrm{CH}_{3}$, $J=7.2), 2.54\left(\mathrm{~s}, 3 \mathrm{H}, \mathrm{CH}_{3}\right), 1.22-1.18\left(\mathrm{t}, 3 \mathrm{H}, \mathrm{CH}_{2} \underline{\mathrm{CH}}_{3}\right.$, $J=7.6)$. MS m/z (\%): $404\left(\mathrm{M}^{+}{ }^{+}-\mathrm{C}_{2} \mathrm{H}_{4} \mathrm{~S} ; 1.3\right), 389$ (3.5), 369 (100), 354 (12.6), 339 (10.3), 77 (9.0), 51 (2.4). Anal. Calcd. for $\mathrm{C}_{23} \mathrm{H}_{21} \mathrm{ClN}_{6} \mathrm{OS}$ (464.97): C 59.41; $\mathrm{H} 4.55 ; \mathrm{Cl} 7.62 ; \mathrm{N}$ 18.07; S 6.90. Found: C 59.39; H 4.53; Cl 7.64; N 18.11; S 6.89 .

$N^{\prime}$-((5-Chloro-3-methyl-1-phenyl-1H-pyrazol-4-yl) methylene)-2-cyano-2-(4-hydroxy-1,3-dithiol-2-ylidene) acetohydrazide 29

To a stirred solution of potassium hydroxide $(0.56 \mathrm{~g}$, $0.01 \mathrm{~mol})$ in DMF $(20 \mathrm{ml})$, compound 3 (3.01 g, $0.01 \mathrm{~mol})$ was added. After stirring for $30 \mathrm{~min}$, the resulting mixture was cooled at $10{ }^{\circ} \mathrm{C}$ in an ice bath, and then, $\mathrm{CS}_{2}(0.6 \mathrm{ml}$, $0.01 \mathrm{~mol}$ ) was added slowly. Stirring was persistent for $12 \mathrm{~h}$ at ambient temperature, and then, chloroacetyl chloride $(0.8 \mathrm{ml}, 0.01 \mathrm{~mol})$ and/or ethyl chloroacetate $(1.07 \mathrm{ml}$, $0.01 \mathrm{~mol})$ were added and stirring was persistent for additional $6 \mathrm{~h}$. The reaction mixture was acidified with cold dilute acetic acid. The separated solid was filtered off, washed several times with cold water, and recrystallized from toluene to give 29 as brown crystals; m.p.: $254{ }^{\circ} \mathrm{C}$, yield: 58\%. IR (KBr): $3424(\mathrm{OH}), 3254(\mathrm{NH}), 2923,2851$ $\left(\mathrm{CH}_{3}\right), 2205(\mathrm{C} \equiv \mathrm{N}), 1620$ (br.s) $(\mathrm{C}=\mathrm{O}), 1603(\mathrm{C}=\mathrm{N}) \mathrm{cm}^{-1}$. ${ }^{1} \mathrm{H}$ NMR (400 MHz, DMSO-d 6 ) $\delta$ (ppm): 11.83 (s, 1H, OH, exchangeable with $\left.\mathrm{D}_{2} \mathrm{O}\right), 9.89(\mathrm{~s}, 1 \mathrm{H}, \mathrm{NH}$, exchangeable with $\left.\mathrm{D}_{2} \mathrm{O}\right), 8.63(\mathrm{~s}, 1 \mathrm{H}, \mathrm{HC}=\mathrm{N}), 7.64-7.46(\mathrm{~m}, 5 \mathrm{H}, \mathrm{Ar}-\mathrm{H})$, 6.69 (s, 1H, HC=C), $2.43\left(\mathrm{~s}, 3 \mathrm{H}, \mathrm{CH}_{3}\right) . \mathrm{MS} \mathrm{m} / \mathrm{z}(\%): 416$ (M. ${ }^{+}-1$; 5.3), 399 (10.2), 375 (15.4), 218 (30.1), 200 (100), 77 (29.5). Anal. Calcd. for $\mathrm{C}_{17} \mathrm{H}_{12} \mathrm{ClN}_{5} \mathrm{O}_{2} \mathrm{~S}_{2}$ (417.89): $\mathrm{C}$ 48.86; H 2.89; Cl 8.48; N 16.76; S 15.34. Found: C 48.84; H 2.90; Cl 8.45; N 16.77; S 15.36 . 


\section{Cytotoxicity and antitumor evaluation}

\section{Materials and methods}

Cell lines Hepatocellular carcinoma (HepG-2) and mammary gland (MCF-7) were obtained from ATCC via Holding company for biological products and vaccines (VACSERA), Cairo, Egypt.

Chemical reagents The reagents RPMI-1640 medium, MTT, and DMSO (sigma co., St. Louis, USA), Fetal Bovine serum (GIBCO, UK).

Doxorubicin was used during comparison as a standard anticancer drug.

MTT assay The different cell lines mentioned above were used to determine the inhibitory effects of compounds on cell growth using the MTT assay [20, 21]. This colorimetric test is based on the change of the yellow tetrazolium bromide (MTT) to a purple formazan derivative by mitochondrial succinate dehydrogenase in viable cells. The cells were cultured in RPMI-1640 medium with $10 \%$ fetal bovine serum. Antibiotics added were $100 \mathrm{units} / \mathrm{ml}$ penicillin and $100 \mu \mathrm{g} /$ $\mathrm{ml}$ streptomycin at $37{ }^{\circ} \mathrm{C}$ in a $5 \% \mathrm{CO}_{2}$ incubator. The cells were seeded in a 96-well plate at a density of $\left(1.0 \times 10^{4}\right.$ cells/ well) at $37{ }^{\circ} \mathrm{C}$ for $48 \mathrm{~h}$ under $5 \% \mathrm{CO}_{2}$. After incubation, the cells were treated with various concentrations of compounds and put in the incubator for $24 \mathrm{~h}$; then, $20 \mu \mathrm{l}$ of MTT solution at $5 \mathrm{mg} / \mathrm{ml}$ was added and incubated for $4 \mathrm{~h}$. DMSO $(100 \mu \mathrm{l})$ was added into each well to dissolve the purple formazan formed. At $570 \mathrm{~nm}$ absorbance, the colorimetric assay was measured and recorded using a plate reader (EXL 800, USA):

Calculation of the relative cell viability (\%)

$=(A$ of treated samples $/ A$ of untreated sample $) \times 100$.

\section{Antioxidant assay}

Antioxidant activity screening assay; ABTS method Determinations of antioxidant activities were estimated from the bleaching of ABTS-derived radical cations. The radical cation was derived from ABTS [2,2'-azino-bis(3-ethyl benzothiazoline-6-sulfonic acid)] was prepared by reaction of ABTS $(60 \mu \mathrm{l})$ with $\mathrm{MnO}_{2}(3 \mathrm{ml}, 25 \mathrm{mg} / \mathrm{ml})$ in phosphate buffer solution $(10 \mu \mathrm{M}, \mathrm{pH} 7,5 \mathrm{ml})$. After the solution was shaking for $3 \mathrm{~min}$, centrifuged and filtered, the Absorbance $A_{\text {(control) }}$ of the resulting ABTS radical solution (green-blue) was recorded at $\lambda_{\max } 734 \mathrm{~nm}$. Upon the addition of $(20 \mu \mathrm{l}$ of $1 \mathrm{mg} / \mathrm{ml}$ ) solution of the tested sample in spectroscopic grade $\mathrm{MeOH} /$ buffer $(1: 1 v / v)$ to the ABTS solution, the absorbance $A_{\text {(test) }}$ was measured. The decrease in the absorbance is expressed as \% inhibition which calculated from this equation [22]:
$\%$ Inhibition $=\left[A_{(\text {control })}-A_{(\text {test })} / A_{(\text {control })}\right] \times 100$,

where the standard antioxidant (positive control) was ascorbic acid solution $(20 \mu \mathrm{l}, 2 \mathrm{mM})$ and blank sample was carried out by solvent without ABTS.

Antioxidant activity screening assay for erythrocyte hemolysis Rats' blood was obtained by cardiac puncture then collected in heparinized tubes. Separation of erythrocytes from plasma was occurred and the buffy coat was washed three times with ten volumes of $0.15 \mathrm{M} \mathrm{NaCl}$. The erythrocytes were centrifuged in the last wash at $2500 \mathrm{rpm}$ for $10 \mathrm{~min}$ to obtain a constantly packed cell preparation. In this assay [23], erythrocyte hemolysis was mediated by peroxyl radicals. The erythrocytes were suspended in phosphate buffered saline (PBS) $\mathrm{pH}$ 7.4. The suspension $(10 \%)$ was added to the same volume of $200 \mathrm{mM}$ 2,2'-azobis(2-amidinopropane)dihydrochloride (AAPH) solution (in PBS) containing samples to be tested at various concentrations. The reaction mixture was shaken gently while being incubated at $37{ }^{\circ} \mathrm{C}$ for $2 \mathrm{~h}$. Then diluted with eight volumes of PBS and centrifuged at $2500 \mathrm{rpm}$ for $10 \mathrm{~min}$. The absorbance $A$ of the supernatant was recorded at $540 \mathrm{~nm}$. Similarly, the reaction mixture was treated with eight volumes of distilled water to achieve complete hemolysis, and the absorbance $B$ of the supernatant obtained after centrifugation was recorded at $540 \mathrm{~nm}$ :

Calculation of the percentage hemolysis $=(1-A / B) \times 100 \%$, where the data were indicated mean standard deviation and the positive control was L-ascorbic acid.

\section{Results and discussion}

\section{Chemistry}

Herein, we report the study of the reactions of the requisite 5-chloro-3-methyl-1-phenyl-1 $H$-pyrazole-4-carbaldehyde 1 [24] with cyanoacetohydrazide 2 in different conditions to obtain variant products.

Condensation of pyrazole aldehyde derivative $\mathbf{1}$ with cyanoacetohydrazide $\mathbf{2}$ in boiling absolute ethanol and/or dioxane tolerated the hydrazide-hydrazone derivative 3 . During refluxing compound $\mathbf{3}$ in absolute ethanol containing a few drops of piperidine afforded the diarylidene hydrazine derivative 4 [25] as light brown crystal. The same product 4 was isolated and identified when the reaction executed in refluxing compound $\mathbf{1}$ with cyanoacetohydrazide $\mathbf{2}$ in ethanol containing a few drops of piperidine directly (Scheme 1).

Heterointra cyclization of compound $\mathbf{3}$ via refluxing it with glacial acetic acid for $10 \mathrm{~h}$ to give pyrazolinone derivative $\mathbf{5}$, beside that the amide derivative $\mathbf{6}$ was obtained. 




Scheme 1 Formation and reactions of compound $\mathbf{3}$ under different conditions<smiles>Cc1n[nH]c(Cl)c1/C=[N+](/[O-])NC(=O)CC#N</smiles>

Syn- isomer<smiles>Cc1n[nH]c(Cl)c1/C=N/NC(=O)CC#N</smiles>

Anti-isomer

Fig. 1 Diasteriomeric isomers of compound 3

Thus, alternative synthetic route for pyrazolinone derivative 5 could be solely obtained in one step by the reaction of compound $\mathbf{1}$ with cyanoacetohydrazide $\mathbf{2}$ in boiling absolute ethanol containing a few drops of glacial acetic acid. On the other hand, refluxing of compound $\mathbf{3}$ in dry pyridine afforded 4 and 5 . Unfortunately, refluxing of compound 3 with anhydrous potassium carbonate in dry acetone, the diarylidene hydrazine derivative $\mathbf{4}$, was obtained as the sole product instead of the desired pyrazolo[3,4-c]pyrazole 7 (Scheme 1).

Noteworthy, compound $\mathbf{4}$ was obtained when we used hard base such as piperidine and $\mathrm{K}_{2} \mathrm{CO}_{3}$ and borderline base as pyridine were classified according to HSAB theory (Pearson concept). Meanwhile, in the case of using hard acid as acetic acid, the cyclized pyrazolinone derivatives $\mathbf{5}$ and $\mathbf{6}$ were obtained.
The spectral and microanalytical data for compounds 3-6 were fit with their chemical structures. The structure of $N^{\prime}$-((5-chloro-3-methyl-1-phenyl-1 $H$-pyrazol-4-yl) methylene)-2-cyanoacetohydrazide 3 was elucidated by elemental analysis and spectroscopic data. The IR spectrum showed the appearance of stretching absorption bands for $v_{\mathrm{NH}}$ at $3192,3126 \mathrm{~cm}^{-1,} v_{\mathrm{C}} \equiv_{\mathrm{N}}$ at $2263 \mathrm{~cm}^{-1}$ and $v_{\mathrm{C}=\mathrm{O}}$ at $1681 \mathrm{~cm}^{-1}$. Furthermore, ${ }^{1} \mathrm{H}$ NMR spectrum of $\mathbf{3}$ is in accordance with its proposed structure, as it showed signals for $\mathrm{NH}$, methyl, methylene, methine, and aromatic protons. Compound $\mathbf{3}$ exists as a diasteriomeric mixture (syn/anti) in the ratio 11.5:88.5. The higher ratio of the anti-isomer is due to its higher stability, since it underwent less steric hindrance because of both hetroaryl and cyanoacetamido groups in the opposite sides. The down field value for the signal of $\mathrm{NH}$ proton as well as the upfield value of the signal of imino methine $(\mathrm{CH}=\mathrm{N})$ proton of the anti-isomer may be attributed to extension of the conjugation as a result of coplanarity of hetroaryl group and $-\mathrm{NH}-\mathrm{CO}-\mathrm{CH}_{2} \mathrm{CN}$ moiety, as shown in Fig. 1.

The mechanistic scenarios for the formation of compounds 4-6 are illustrated, as shown in Scheme 2.

The ${ }^{1} \mathrm{H}$ NMR spectrum of compound 6 in DMSO solution revealed the presence of four peaks exchangeable with 
Scheme 2 Mechanistic pathways to compounds 4-6

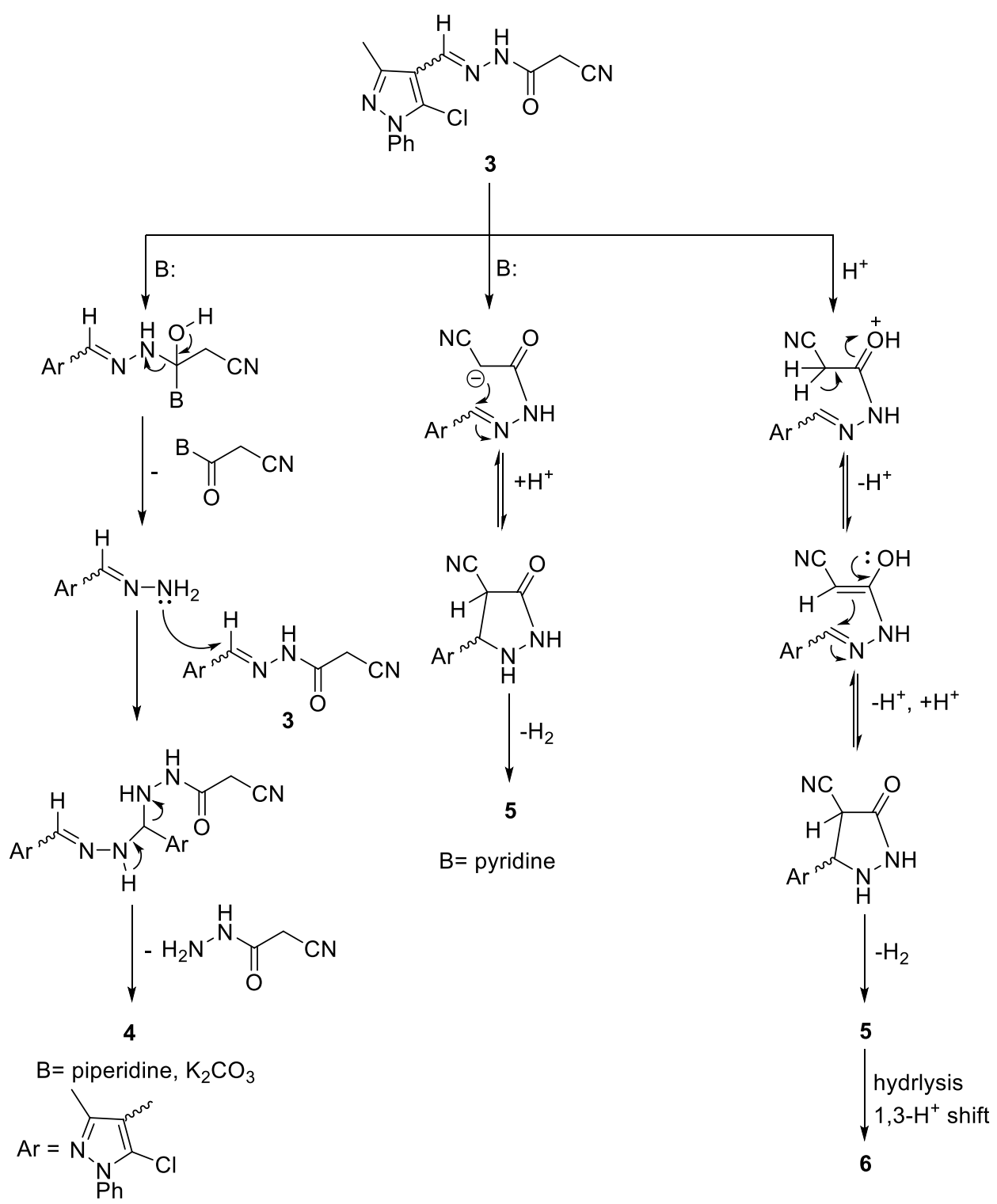<smiles></smiles>

$51 \%$<smiles>Cc1n[nH]c(Cl)c1C1=NN=C2OC[O+]=C21</smiles>

$49 \%$
Fig. 2 Diastereomeric mixture of compound 6

$\mathrm{D}_{2} \mathrm{O}$ at $\delta=13.31,12.20,10.65$, and 10.40 ppm corresponding to $\mathrm{OH}, \mathrm{NH}_{2}, \mathrm{NH}$, and $\mathrm{NH}_{2}$, respectively. ${ }^{1} \mathrm{H}$ NMR of 6 revealed its existence as a diasteromeric mixture in the ratio of 51:49. The down field shifts of $\mathrm{OH}$ and $\mathrm{NH}_{2}$ protons are due to intramolecular chelated $\mathrm{H}$-bond in six-membered ring (Fig. 2).

Focusing on reactivity of $\beta$-ketonitriles towards some nucleophilic and electrophilic agents led us to explore the utilization of the hydrazide-hydrazone derivative $\mathbf{3}$ as a precursor to construct more heterocycles.

Therefore, compound $\mathbf{3}$ was allowed to react with hydrazine hydrate to constructing pyrazole derivative $\mathbf{8}$, as described in the literature [26, 27]. However, its reaction with hydrazine hydrate produced the diarylidene hydrazine derivative $\mathbf{4}$ instead of $\mathbf{8}$ (Scheme 3).

Plausible mechanistic pathways to demonstrate the formation of compound $\mathbf{4}$ is illustrated in Scheme 4. The pathway b was extrapolated from the anterior mechanism.

The proclivity of $\mathbf{3}$ towards some electrophilic reagents such as aromatic aldehydes, heterocyclic ketone, aliphatic 
Scheme 3 Hydrazinolysis of compound $\mathbf{3}$

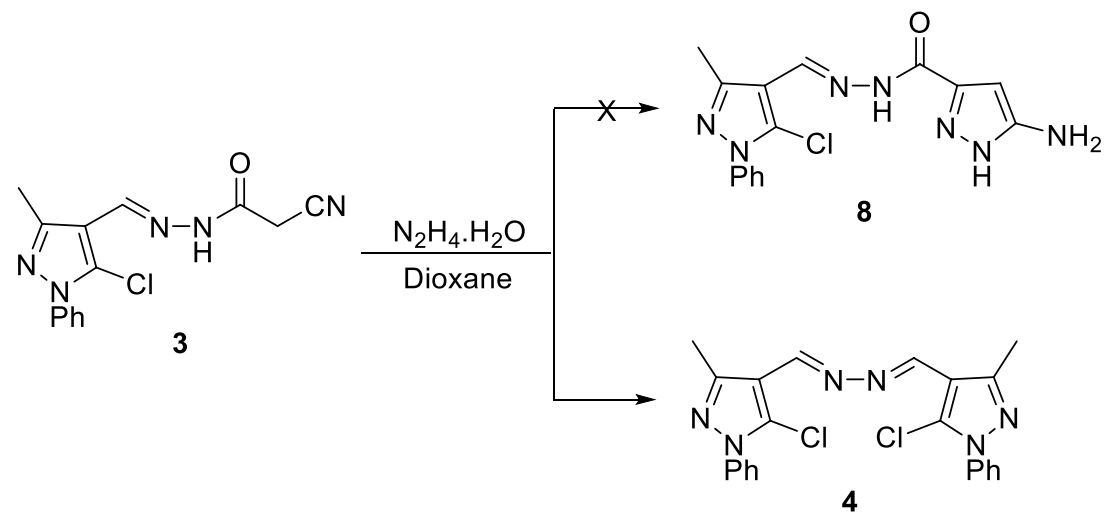

Scheme 4 Mechanistic illustration of $\mathbf{4}$ formation

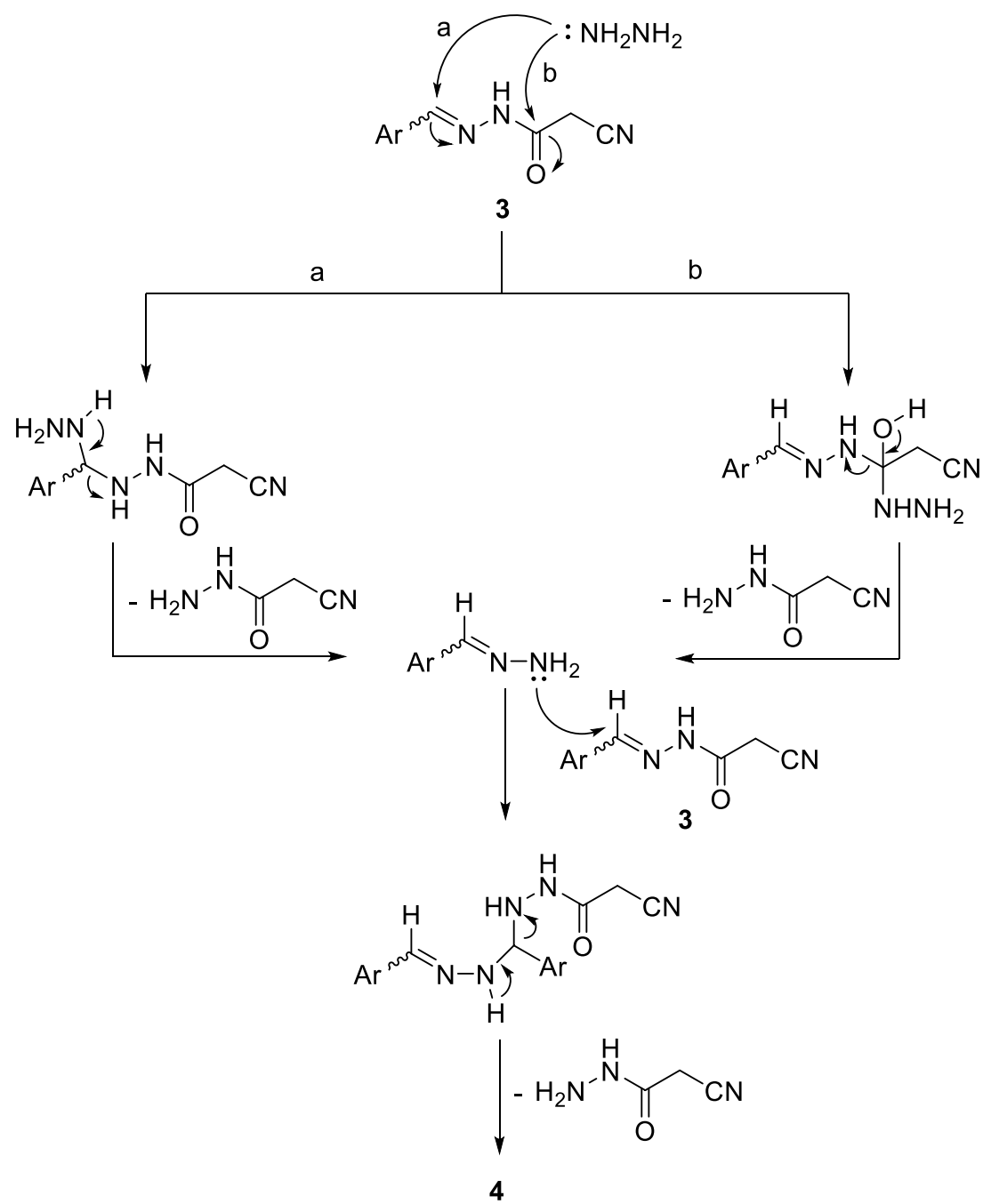

ketone, arylidene malononitrile, and phenyl isothiocyanate was also investigated as follow in Scheme 5.

In our previous work $[17,18]$, when $\beta$-ketonitrile was refluxed with $p$-nitrobenzaldehyde in boiling ethanol in the presence of piperidine, the corresponding condensation product 9 was afforded (Scheme 5). Meanwhile, in this study, the diarylidene hydrazine derivative $\mathbf{4}$ was obtained instead. As an alternative way, when a harder base such as sodium ethoxide used at room temperature instead of piperidine, the reaction was smoothly achieved to afford 9 (Scheme 5). The IR spectrum of compound 9 exhibited stretching absorption bands at $2210 \mathrm{~cm}^{-1}(\mathrm{C} \equiv \mathrm{N})$ and 1680 , 


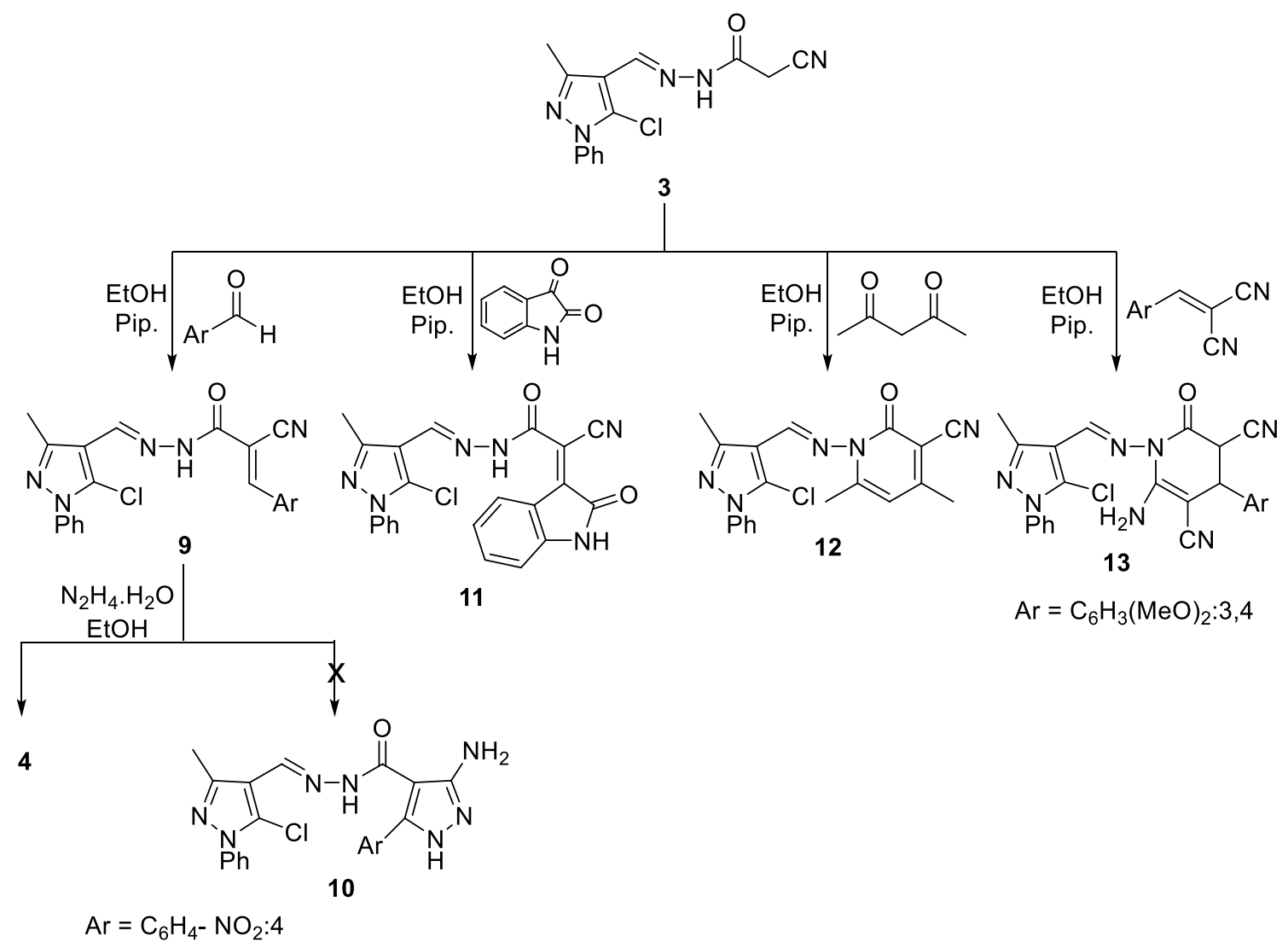

Scheme 5 Synthetic pathway to compounds 9-13

Fig. 3 Lactam-lactim tautomers of compound 9<smiles></smiles>

$39 \%$

$61 \%$ yield as dark brown crystals (Scheme 5). The structure 11 was substantiated from the microanalytical and spectroscopic data.

The synthesis of 4,6-dimethyl pyridinone derivative $\mathbf{1 2}$ was commenced from the reaction of cyanoacetamide $\mathbf{3}$ with acetylacetone in ethanolic solution containing drops of piperidine. As a proof for the proposed structure of $12,{ }^{1} \mathrm{H}$ NMR spectrum of $\mathbf{1 2}$ showed characteristics singlet peaks for $\mathrm{HC}=\mathrm{N}, \mathrm{CH}_{\text {pyridinone }}, \mathrm{CH}_{3 \text { pyrazole }}$, and two $\mathrm{CH}_{3 \text { pyridinone }}$ at $\delta=8.76,6.39,2.47,2.346$, and 2.343 ppm, respectively, besides multiplet peak for $\mathrm{Ph}$ at $\delta=7.62-7.51 \mathrm{ppm}$.

Heating a mixture of compound 3 with 2-(3,4-dimethoxybenzylidene)malononitrile in absolute ethanol containing a catalytic amount of piperidine afforded 6-amino-2-oxo1,2,3,4-tetrahydropyridine derivative 13 (Scheme 5). The 


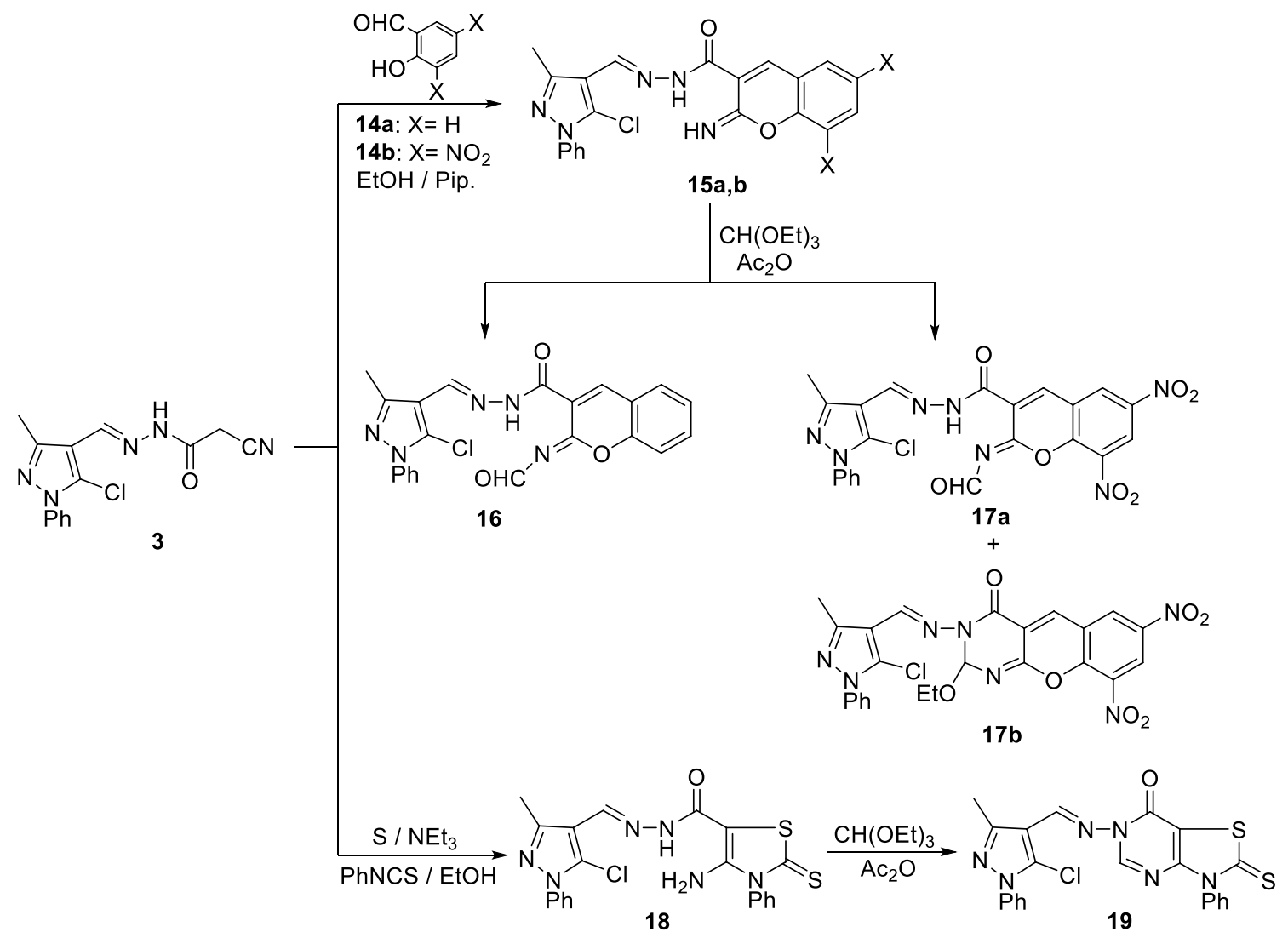

Scheme 6 Synthetic pathway to compounds 15a,b-19

proposed structure of $\mathbf{1 3}$ was confirmed by spectral and elemental analyses.

Following the same literately reported a synthesis of iminocoumarin derivatives $[17,18]$, herein, novel iminocoumarin derivatives were built upon refluxing compound 3 with an ethanolic solution of salicylaldehyde and/or 3,5-dinitrosalicylaldehyde $\mathbf{1 4 a , b}$ (1:1 molar ratio) in the presence of a catalytic amount of piperidine.(Scheme 6).

The structures of iminocoumarin derivatives 15a,b were unambiguously ascertained on the basis of analytical and spectroscopic data. For example, the IR spectrum of $15 \mathbf{a}$ displayed $v_{\mathrm{NH}}$ at $3312 \mathrm{~cm}^{-1}, v_{\mathrm{C}=\mathrm{O}}$ at $1682 \mathrm{~cm}^{-1}$ and $v_{\mathrm{C}=\mathrm{N}}$ at $1632 \mathrm{~cm}^{-1}$. At the same time, the IR spectrum of $15 \mathrm{~b}$ displayed $v_{\mathrm{NH}}$ at $3206 \mathrm{~cm}^{-1}, v_{\mathrm{C}=\mathrm{O}}$ at $1683 \mathrm{~cm}^{-1}$, $v_{\mathrm{C}=\mathrm{N}}$ at $1619 \mathrm{~cm}^{-1}$ and $v_{\mathrm{NO} 2}$ at 1550 and $1339 \mathrm{~cm}^{-1}$, it is also worthy to mentioned that IR of both compounds showed the absence of a stretching absorption band for nitrile group.

Afterwards, compounds $\mathbf{1 5 a , b}$ were individually refluxed with triethyl orthoformate in the presence of distilled acetic anhydride to afforded $\mathbf{1 6}$ and $\mathbf{1 7 a} \mathbf{a}, \mathbf{b}$ (Scheme 6).

Characterizations of the newly synthesized compounds 16, 17a,b were based on spectral and elemental analyses.
The IR spectrum of compound 16 refers to the existence of an aldehydic carbonyl group at $1710 \mathrm{~cm}^{-1}$. Moreover, the ${ }^{1} \mathrm{H}$ NMR spectrum indicated the presence of a singlet peak at $\delta 8.83 \mathrm{ppm}$ (aldehydic proton).

For compounds 17a,b, the IR spectrum showed the presence of a stretching absorption band for the carbonyl groups at 1719 and $1678 \mathrm{~cm}^{-1}$. As well, the ${ }^{1} \mathrm{H}$ NMR spectrum revealed the existence of a mixture of the two compounds $\mathbf{1 7} \mathbf{a}, \mathbf{b}$ due to the presence of methine proton as singlet at $\delta 5.83 \mathrm{ppm}$ and ethoxy protons as quartet and triplet at 3.66-3.56 and 1.01 ppm, respectively. Besides, the presence of aldehydic proton as singlet peak at $\delta 9.03 \mathrm{ppm}$.

Refluxing a three component, an ethanolic mixture of $\mathbf{3}$ with phenyl isothiocyanate and elemental sulfur afforded 2-thioxo-2,3-dihydrothiazole derivative $\mathbf{1 8}$ under basic condition $\left(\mathrm{Et}_{3} \mathrm{~N}\right)$ (Scheme 6).

The structure of $\mathbf{1 8}$ was unequivocally proven via investigating the IR spectrum which exhibited the absence of cyano group. Furthermore, ${ }^{1} \mathrm{H}$ NMR displayed broad singlet at $\delta 6.31$ ppm exchangeable with $\mathrm{D}_{2} \mathrm{O}$ corresponding to $\mathrm{NH}_{2}$ protons.

2-Thioxo-2,3-dihydrothiazolo[4,5- $d]$ pyrimidin-7(6H)-one derivative 19 was furnished when 18 was subjected to react 
Scheme 7 Synthetic pathway to compounds 21-23

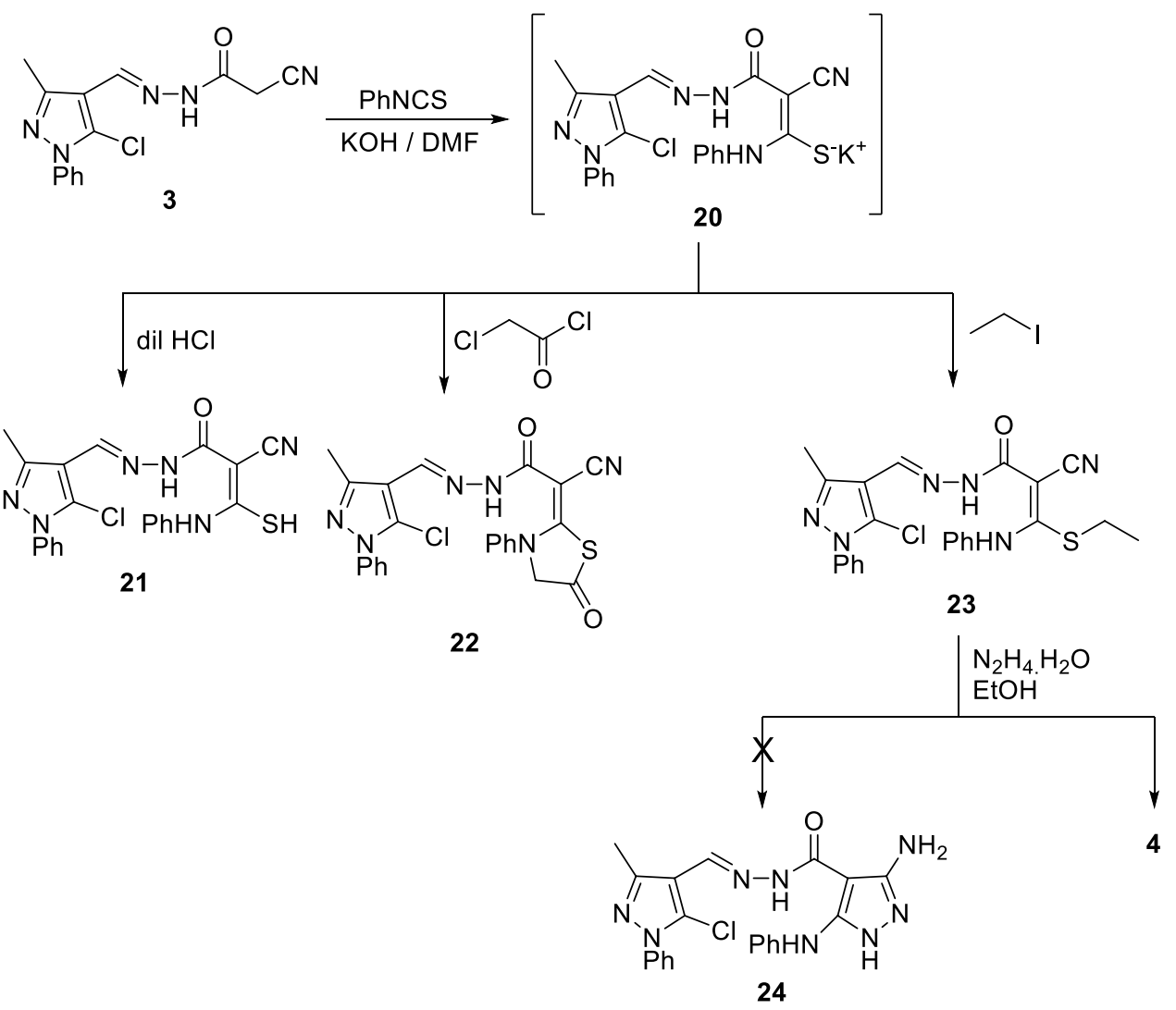

with triethyl orthoformate in the presence of distilled acetic anhydride (Scheme 6). The proposed structure of $\mathbf{1 9}$ was confirmed by spectral and elemental analyses. In particular, ${ }^{1} \mathrm{H}$ NMR spectrum of $\mathbf{1 9}$ showed a characteristic peak for $\mathrm{CH}_{\text {pyrimidinone }}$ at $\delta 9.31 \mathrm{ppm}$ and the absence of peak of $\mathrm{NH}_{2}$ protons.

The reactivity of the active methylene moiety of compound $\mathbf{3}$ was investigated. Thus, when compound $\mathbf{3}$ was allowed to react with phenyl isothiocyanate in dry DMF containing a catalytic amount of potassium hydroxide at room temperature [18, 19], it yielded the nonisolable intermediate potassium sulfide salt $\mathbf{2 0}$, followed by acidification with dilute $\mathrm{HCl}$ to isolate compound $\mathbf{2 1}$ in good yield (Scheme 7). The microanalytical and spectroscopic data were in agreement with the proposed structure 21 .

Otherwise, treatment of the nonisolable potassium sulfide salt $\mathbf{2 0}$ in situ with chloroacetyl chloride afforded 1,3-thiazolidin-5-one derivative 22 (Scheme 7). Supposedly, the reaction mechanism is assumed to proceed via $\mathrm{S}$-acylation followed by closure of the thiazole ring upon $\mathrm{N}$-alkylation of the remaining $\mathrm{CH}_{2} \mathrm{Cl}$ group to award thiazolidinone derivative 22. Elemental analyses and spectral data were in favor of this proposed 1,3-thiazolidin-5-one structure. The IR spectrum of $\mathbf{2 2}$ showed absorption band at $1745 \mathrm{~cm}^{-1}$ due to carbonyl group of the thiazolidinone ring. The structure of 22 was also ascertained from ${ }^{1} \mathrm{H}$ NMR spectrum of 22 which revealed the presence of a singlet peak at $\delta=3.89 \mathrm{ppm}$ corresponding to $\mathrm{CH}_{2}$ protons of the thiazolidinone ring.

Moreover, curing of the nonisolable potassium sulfide salt 20 with ethyl iodide afforded $N^{\prime}$-((5-chloro-3-methyl-1phenyl-1 $H$-pyrazol-4-yl)methylene)-2-cyano-3-(ethylthio)3-(phenylamino)acrylohydrazide 23 (Scheme 7).

Unfortunately, when the novel ketene $N, S$-acetal $\mathbf{2 3}$ was refluxed with hydrazine hydrate in ethanol, the sulfur-free compound which was characterized as the diarylidene hydrazine derivative $\mathbf{4}$ was obtained as the sole product instead of the desired pyrazole derivative 24 [19]. No clue for the formation of 3-aminopyrazole derivative $\mathbf{2 4}$ was detected neither from spectroscopic nor elemental analyses (Scheme 7).

Finally, compound $\mathbf{3}$ with carbon disulfide in the presence of potassium hydroxide in DMF afforded the intermediate dipotassium dithiolate salts $\mathbf{2 5}$ which in situ underwent S-acylation with chloroacetyl chloride and/or S-alkylation with ethyl chloroacetate as different intermediates $\mathbf{2 6}$ and $\mathbf{2 7}$, respectively. In both cases, the 4-oxo-1,3-dithiolane derivative 28 was anticipated. However, unexpectedly, its enol tautomer 29 was obtained instead. The chemical structure of 29 was supported on the basis of elemental analysis and spectral data (Scheme 8). 
Scheme 8 Synthetic pathways to 4-hydroxy-1,3-dithiole derivative $\mathbf{2 8}$

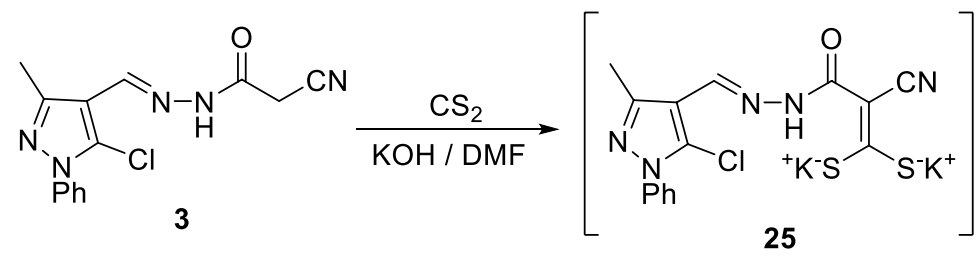

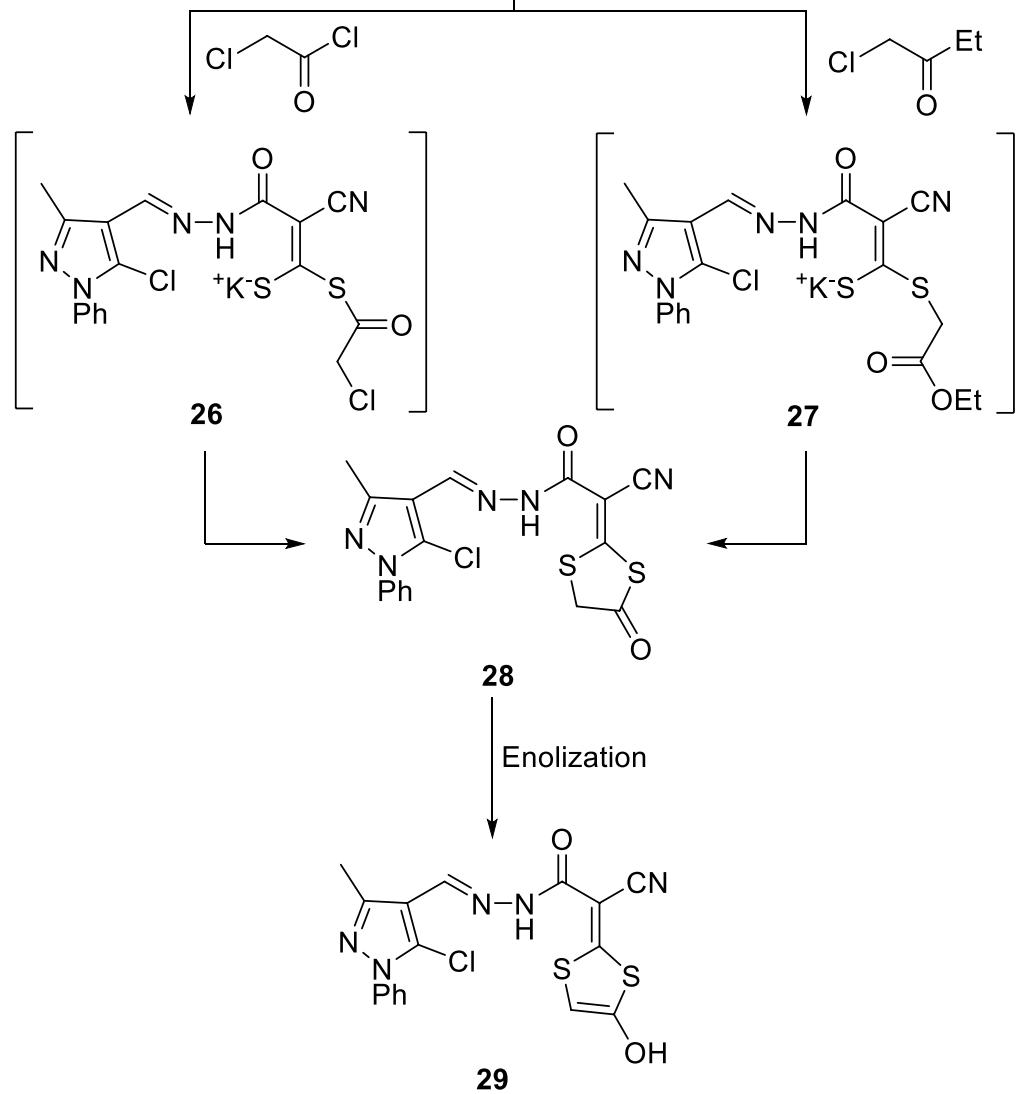

\section{Pharmacology}

\section{Cytotoxicity and antitumor evaluation}

Out of the newly synthesized compounds, 14 analogs were selected to be evaluated for their in-vitro anticancer effects via the standard MTT method [20,21], against a panel of two human tumor cell lines; (HepG2) hepatocellular carcinoma and (MCF-7) mammary gland breast cancer. Doxorubicin was used during comparison as a standard anticancer drug. The results of cytotoxic activity of compounds are summarized in Table 1 and Fig. 4.

The obtained results revealed that the tested compounds, namely, 4, 6, 9, 11, 13, 15a, 15b, 16, 18, 19, 21, 22, 23, and 29 exhibited variable degrees of inhibitory activity towards the two tested human tumor cell lines. As for activity against HepG2, the very strongest cytotoxic activity was exhibited by compounds $\mathbf{1 3}$ and $\mathbf{2 9}$ which showed the percentage viability $\mathrm{IC}_{50}$ at $6.48 \pm 0.6$ and $9.16 \pm 0.9 \mu \mathrm{M}$, respectively. Whereas, the strongest cytotoxic activity was exhibited by compounds $\mathbf{6 , 1 1}$, and 22 which showed the percentage viability $\mathrm{IC}_{50}$ at $15.90 \pm 1.4,19.02 \pm 1.6$, and $11.79 \pm 1.2 \mu \mathrm{M}$, respectively. Meanwhile, compounds 15a, $15 b, 4$, and 18 showed moderate activity, while other compounds showed weak activities. On the other hand, the activity against MCF-7 cell line revealed that compounds 13 and 29 have the very highest percentage viability $\mathrm{IC}_{50}$ at $5.48 \pm 0.4$ and $8.26 \pm 0.7 \mu \mathrm{M}$, respectively. Both compounds 6 and 22 showed the strongest activities. Whereas, compounds $4,11,15 \mathrm{a}, 15 \mathrm{~b}$, and 18 showed moderate activities. Meanwhile, the other tested compounds exhibited weak activities.

Structure activity relationships (SARs) By comparing the cytotoxicity assessment of the reported compounds, in this study, we conclude the following essential structural features for activity enhancement. 
Table 1 Cytotoxic activity of some new compounds against human tumor cells

\begin{tabular}{lrr}
\hline Compounds & \multicolumn{2}{l}{ In-vitro cytotoxicity $\mathrm{IC}_{50}(\mu \mathrm{M})$} \\
\cline { 2 - 3 } & \multicolumn{1}{c}{ HepG2 } & \multicolumn{1}{c}{ MCF-7 } \\
\hline DOX & $4.50 \pm 0.3$ & $4.17 \pm 0.2$ \\
4 & $43.41 \pm 2.8$ & $48.03 \pm 3.0$ \\
6 & $15.90 \pm 1.4$ & $12.71 \pm 1.1$ \\
9 & $52.96 \pm 3.4$ & $61.34 \pm 4.1$ \\
11 & $19.02 \pm 1.6$ & $23.34 \pm 1.8$ \\
13 & $6.48 \pm 0.6$ & $5.48 \pm 0.4$ \\
$15 \mathrm{a}$ & $31.74 \pm 2.3$ & $35.82 \pm 2.5$ \\
$15 \mathrm{~b}$ & $36.22 \pm 2.5$ & $40.85 \pm 2.8$ \\
16 & $69.53 \pm 3.9$ & $72.82 \pm 4.5$ \\
18 & $24.86 \pm 1.9$ & $29.28 \pm 2.2$ \\
19 & $64.78 \pm 3.7$ & $58.96 \pm 3.6$ \\
21 & $83.45 \pm 4.8$ & $91.75 \pm 5.2$ \\
22 & $11.79 \pm 1.2$ & $18.36 \pm 1.5$ \\
23 & $74.63 \pm 4.2$ & $51.40 \pm 3.4$ \\
29 & $9.16 \pm 0.9$ & $8.26 \pm 0.7$ \\
\hline
\end{tabular}

$\mathrm{IC}_{50}(\mu \mathrm{M})$ : 1-10 (very strong), 11-20 (strong), 21-50 (moderate), 51-100 (weak) and above 100 (non-cytotoxic)

DOX Doxorubicin

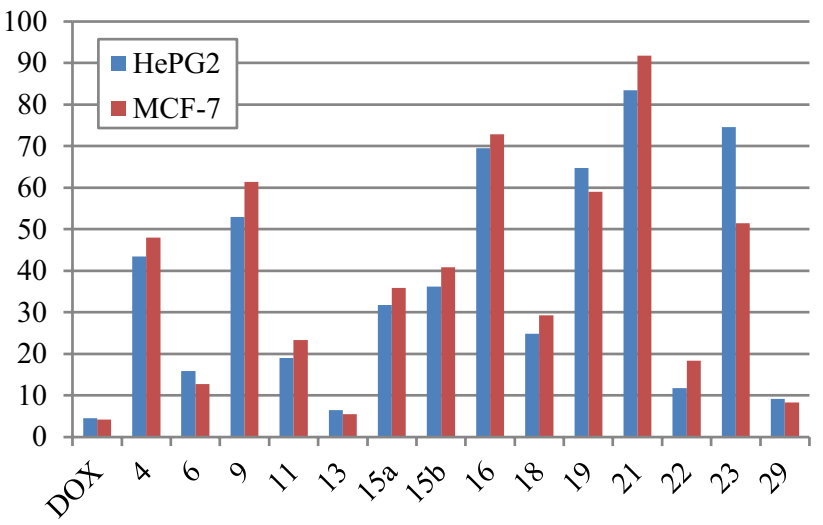

Fig. 4 Cytotoxic activity of some new compounds against human tumor cells

(1) The presence of pyrazole skeleton is necessary for the broadening of cytotoxic activity towards both cell lines (HepG2 and MCF-7).

(2) Since compound $\mathbf{1 3}$ has a remarkable potency closer to doxorubicin against both cell lines (HepG2 and MCF7) which may be attributed to the presence of 3,4-dihydropyridinone moiety, beside incorporating of amino and two methoxy groups (electron donating groups).

(3) 4-Hydroxy-1,3-dithiole ring in $\mathbf{2 9}$ enhances the antitumor activity against both cell lines.

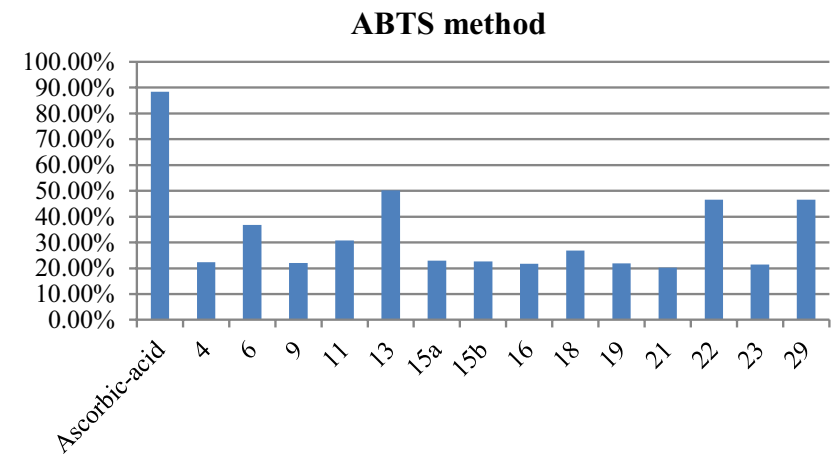

Fig. 5 Antioxidant activity of some new compounds by ABTS method

\section{Erythrocyte haemolysis}



Fig. 6 Antioxidant activity of some new compounds by erythrocyte hemolysis method

Table 2 Antioxidant activity of some new compounds by ABTS method

\begin{tabular}{lll}
\hline Method & \multicolumn{2}{l}{$\begin{array}{l}\mathrm{ABTS} \\
\mathrm{Abs}_{(\mathrm{control})}-\mathrm{Abs}_{(\text {(test })} / \mathrm{Abs}_{\text {(control) }} \times 100\end{array}$} \\
\cline { 2 - 3 } Compounds & Absorbance of samples & $\%$ Inhibition \\
\hline Control of ABTS & 0.525 & - \\
Ascorbic acid & 0.061 & 88.4 \\
4 & 0.408 & 22.3 \\
6 & 0.332 & 36.8 \\
9 & 0.409 & 22.1 \\
11 & 0.363 & 30.8 \\
13 & 0.262 & 50.1 \\
$15 \mathrm{a}$ & 0.404 & 23.0 \\
$15 \mathrm{~b}$ & 0.406 & 22.7 \\
16 & 0.411 & 21.7 \\
18 & 0.384 & 26.8 \\
19 & 0.410 & 21.9 \\
21 & 0.419 & 20.2 \\
22 & 0.281 & 46.5 \\
23 & 0.412 & 21.5 \\
29 & 0.281 & 46.5 \\
\hline
\end{tabular}


Table 3 Antioxidant activity of some new compounds by erythrocyte hemolysis method

\begin{tabular}{|c|c|c|}
\hline \multirow{2}{*}{$\begin{array}{l}\text { Method } \\
\text { Compounds }\end{array}$} & \multicolumn{2}{|c|}{$\begin{array}{l}\text { Erythrocyte hemolysis } \\
A / B \times 100\end{array}$} \\
\hline & $\begin{array}{l}\text { Absorbance of } \\
\text { samples }\end{array}$ & $\%$ Hemolysis \\
\hline Absorbance of $\mathrm{H}_{2} \mathrm{O}(B)$ & 0.850 & - \\
\hline Vit-C & 0.031 & 3.64 \\
\hline 4 & 0.031 & 3.64 \\
\hline 6 & 0.033 & 3.88 \\
\hline 9 & 0.039 & 4.58 \\
\hline 11 & 0.034 & 4.00 \\
\hline 13 & 0.031 & 3.64 \\
\hline $15 \mathrm{a}$ & 0.043 & 5.05 \\
\hline $15 b$ & 0.042 & 4.94 \\
\hline 16 & 0.034 & 4.00 \\
\hline 18 & 0.046 & 5.41 \\
\hline 19 & 0.038 & 4.47 \\
\hline 21 & 0.032 & 3.76 \\
\hline 22 & 0.034 & 4.00 \\
\hline 23 & 0.046 & 5.41 \\
\hline 29 & 0.031 & 3.64 \\
\hline
\end{tabular}

(4) Cyclization of compound $\mathbf{3}$ into 4,5-dihydropyrazolone derivative 6 incorporating amide group in position 4 enhances the antitumor activity against both cell lines.

(5) Introduction of indolin-2-one moiety into compound 11 increases the antitumor activity against $\mathrm{HepG} 2$ cell line.

(6) Compound $\mathbf{2 2}$ is more potent than $\mathbf{2 1}$ and $\mathbf{2 3}$ which may be due to conversion of compound $\mathbf{3}$ into 1,3-thiazolidin-5-one moiety.

(7) Compound $\mathbf{1 6}$ is less potent than compound 15a which may be attributed to the conversion of the iminocoumarin into $N$-formyl moiety. In addition, compound 19 is less potent than compound $\mathbf{1 8}$ which may be attributed to the conversion of 4-amino thiazol-2-thione into 2-thioxo-2,3-dihydrothiazolo[4,5-d]pyrimidin-7(6H)one moiety. As a conclusion, it is obvious that in these two cases, disappearance of $\mathrm{NH}$ and $\mathrm{NH}_{2}$ diminishes the potency which could be refused to declining in hydrophobicity character.

\section{Antioxidant activity screening}

The newly synthesized compounds were selected and screened for antioxidant activity. Antioxidant effects of the compounds were screened using ABTS assay. Compounds 13, 22, and 29 displayed promising activities. In the meantime, compounds $\mathbf{6}$ and $\mathbf{1 1}$ showed moderate activities.
In case of erythrocytes hemolysis, compounds 4,13 , and 29 proved to exhibit potent antioxidative activity as vitamin C, while compounds $\mathbf{6}, \mathbf{1 1}, \mathbf{1 6}, \mathbf{2 1}$, and $\mathbf{2 2}$ showed very high activities.

From Tables 2 and 3 and Figs. 5 and 6 and the abovementioned results, we may include the following structure activity relationships (SARs).

(1) The presence of 3,4-dihydropyridinone, 1,3-thiazolidin-5-one and 4-hydroxy-1,3-dithiole moieties in compounds $\mathbf{1 3}, \mathbf{2 2}$, and $\mathbf{2 9}$, respectively, besides the incorporation of pyrazole ring bearing methyl, phenyl groups, and chlorine atom proved to be vital for remarkable potential inhibitory antioxidant activity using ABTS method.

(2) Introduction of heterocyclic moieties as in compounds 4, 13, and 29 enhanced the antioxidant activity in erythrocytes hemolysis to the same extent of vitamin C. Those compounds are currently under more evaluation as a potential candidate drugs.

Literately, some previous reports showed that structurally similar but they are different in pyrazole moiety which manifested different results in antitumor and antioxidant activities than our results [17, 29].

\section{Conclusion}

The goal of the present study is too utilizing some pyrazole derivatives as scaffold for constructing different heterocyclic moieties on it. As an ultimate goal, those synthesized compounds were screened and evaluated as potent agents against the antitumor and antioxidant activity. The data collection showed clearly that compounds $6,11,13,22$, and 29 displayed promising in-vitro antitumor activity against two cell lines (HepG2 and MCF-7). Among these compounds, compounds 13 and $\mathbf{2 9}$ exhibited broad spectrum of antitumor agent. On the other hand, compounds 13, 22, and 29 exhibited the highest inhibitory antioxidant activity using ABTS method. Meanwhile, in case of erythrocytes hemolysis, compounds 4,13 , and 29 proved to exhibit potent antioxidative activity as vitamin C. Noteworthy, Compounds $\mathbf{1 3}$ and $\mathbf{2 9}$ manifested the highest potency in targeting both antitumor and antioxidant activities.

\section{References}

1. S. Viveka, P. Dinesha, S. Shama, N.K. Naveen, G.K. Lokanath, Nagaraja, RSC Adv. 5, 94786 (2015)

2. H.M. Faidallah, M.M. Al-Mohammadi, K.A. Alamry, K.A. Khan, J. Enzym. Inhib. Med. Chem. 31, 157 (2016) 
3. S. Ghadbeigi, S.N. Ostad, A. Shafiee, M. Amini, Lett. Drug Des. Discov. 12, 754 (2015)

4. S.G. Alegaon, K.R. Alagawadi, M.K. Garg, K. Dushyant, D. Vinod, Bioorg. Chem. 54, 51 (2014)

5. H.V. Chavan, B.P. Bandgar, L.K. Adsul, V.D. Dhakane, P.S. Bhale, V.N. Thakare, V. Masand, Bioorg. Med. Chem. Lett. 23, 1315 (2013)

6. D.C. Malvar, R.T. Ferreira, R.A. de Castro, L.L. de Castro, A.C.C. Freitas, E.A. Costa, I.F. Florentino, J.C.M. Mafra, G.E.P. de Souza, F.A. Vanderlinde, Life Sci. 95, 81 (2014)

7. M. Jyothi, R. Merugu, Int. J. Pharm. Tech. Res. 8, 80 (2015)

8. K.P. Beena, A.A.A. Elkhider, P. Shebina, V. Sindhuja, J. Chem. Pharm. Res. 8, 915 (2016)

9. S. Du, Z. Tian, D. Yang, X. Li, H. Li, C. Jia, C. Che, M. Wang, Z. Qin, Molecules 20(5), 8395 (2015)

10. C.R. Fu, J. Pei, Y. Ning, M. Liu, P.C. Shan, J. Liu, Y.Q. Li, F.Z. Hu, Y.Q. Zhu, H.Z. Yang, Pest Manag. Sci. 70, 1207 (2014)

11. H. Wu, J.-T. Feng, K.-C. Lin, X. Zhang, Molecules 17, 12187 (2012)

12. R. Perez-Fernandez, P. Goya, J. Elguero, Arkivoc 2, 233 (2014)

13. Z. Hajizadeh, A. Maleki, Mol. Catal. 460, 87 (2018)

14. A. Maleki, A.A. Jafari, S. Yousefi, Carbohydr. Polym. 175, 409 (2017)

15. A. Shaabani, M. Seyyedhamzeh, A. Maleki, M. Behnam, F. Rezazadeh, Tetrahedron Lett. 50, 2911 (2009)

\section{Affiliations}

\section{Mahmoud F. Ismail $^{1}$ D . Amira A. El-sayed ${ }^{1}$}

Mahmoud F. Ismail

fawzy2010@sci.asu.edu.eg
16. A.A. Fadda, M.M. Mukhtar, M.R. Hala, Am. J. Org. Chem. 2(2), $32(2012)$

17. M.F. Ismail, G.A. El-sayed, Synth. Commun. 48, 892 (2018)

18. M.R. Mahmoud.;, S.A. Shiba, A.K. El-Ziaty, F.S.M. Abu El-Azm, M.F. Ismail, Synth. Commun. 44, 1094 (2014)

19. M.R. Mahmoud, A.K. El-Ziaty, F.S.M. Abu El-Azm, M.F. Ismail, S.A. Shiba, J. Chem. Res. 37, 80 (2013)

20. T. Mosmann, J. Immunol. Methods 65(1-2), 55 (1983)

21. F. Denizot, R. Lang, J. Immunol. Methods 89(2), 271 (1986)

22. E.A. Lissi, B. Modak, R. Torres, J. Escobar, A. Urzua, Free Radic. Res. 30, 471 (1999)

23. Y. Morimoto, K. Tanaka, Y. Iwakiri, S. Tokuhiro, S. Fukushima, Y. Takeuchi, Biol. Pharm. Bull. 18(10), 1417 (1995)

24. Y.-Q. Cun-Jin, Shi, J. Chem. Crystalloger. 41, 1816 (2011)

25. F.M. Abd El-Latif, M.A. Barsy, E.A. Elrady, M. Hassan, J. Chem. Res. (S) 12, 696 (1999)

26. N. Suryakiran, D. Ramesh, Y. Venkateswarlu, Green Chem. Lett. Rev. 1(1), 73 (2007)

27. T.P. Larry, M.C. Michael, O.M. Florence, Tetrahedron 67, 4601 (2011)

28. M. Rafat, Z. Jonathan, A.M. Abeer, Phosphorus Sulfur Silicon Relat. Elem. 182, 1661 (2007)

29. W.W. Wardakhan, S.M. Sherif, R.M. Mohareb, A.S. Abouzied, Int. J. Org. Chem. 2, 321 (2012)

1 Department of Chemistry, Faculty of Science, Ain Shams University, Cairo, Abbassia 11566, Egypt 